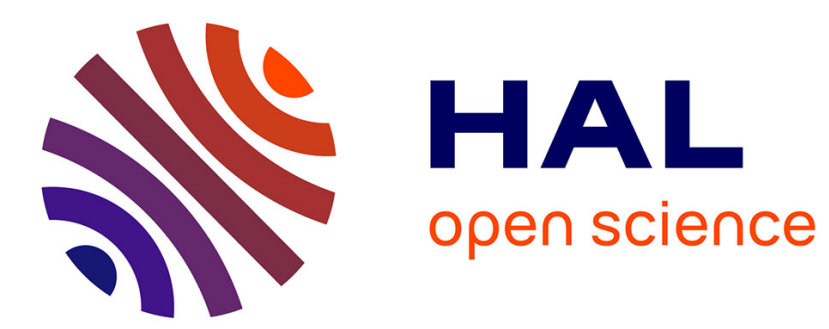

\title{
Uniting observers
}

\author{
Daniele Astolfi, Romain Postoyan, Dragan Nešić
}

\section{To cite this version:}

Daniele Astolfi, Romain Postoyan, Dragan Nešić. Uniting observers. IEEE Transactions on Automatic

Control, 2020, 65 (7), pp.2867-2882. 10.1109/TAC.2019.2933395 . hal-02283837

\section{HAL Id: hal-02283837 \\ https://hal.science/hal-02283837}

Submitted on 11 Sep 2019

HAL is a multi-disciplinary open access archive for the deposit and dissemination of scientific research documents, whether they are published or not. The documents may come from teaching and research institutions in France or abroad, or from public or private research centers.
L'archive ouverte pluridisciplinaire HAL, est destinée au dépôt et à la diffusion de documents scientifiques de niveau recherche, publiés ou non, émanant des établissements d'enseignement et de recherche français ou étrangers, des laboratoires publics ou privés. 


\title{
Uniting Observers
}

\author{
Daniele Astolfi ${ }^{a}$, Romain Postoyan ${ }^{b}$ and Dragan Nešić ${ }^{c}$
}

\begin{abstract}
We propose a framework for designing observers possessing global convergence properties and desired asymptotic behaviours for the state estimation of nonlinear systems. The proposed scheme consists in combining two given continuoustime observers: one, denoted as global, ensures (approximate) convergence of the estimation error for any initial condition ranging in some prescribed set, while the other, denoted as local, guarantees a desired local behaviour. We make assumptions on the properties of these two observers, and not on their structures, and then explain how to unite them as a single scheme using hybrid techniques. Two case studies are provided to demonstrate the applicability of the framework. Finally, a numerical example is presented.
\end{abstract}

Index Terms-Observers, nonlinear systems, hybrid systems, local performances, extended Kalman filters.

\section{INTRODUCTION}

When designing an observer for a dynamical system, we first of all want to ensure that the produced state estimate converges towards the plant state as time grows. We also desire to ensure the following key properties:

(a) (domain of attraction) the convergence should be guaranteed irrespectively of the observer initialization;

(b) (convergence speed) a certain convergence rate should be required for the observer to rapidly generate accurate estimates of the state;

(c) (model robustness) the estimate needs to be accurate even in presence of model uncertainties;

(d) (sensitivity to noise) the quality of the estimation should not be too sensitive to measurement noise.

It is very difficult, if not impossible ${ }^{1}$, to address all these requirements at the same time, in particular when dealing with nonlinear finite-dimensional systems. Hence, the observer design often results in a trade-off between some of the properties listed above. For instance, high-gain observers (HGO), see e.g., [2], or sliding mode observers, see e.g., [3], typically satisfy requirements (a), (b), and (c), but fail in (d). Extended Kalman filters (EKF) on the other hand, see e.g., [4], [5], ensure the properties (b), (c), and (d), but do not guarantee (a) in general. A natural idea to overcome these limitations is therefore that of combining different observers or gains to inherit the "good" properties of each of them. This

${ }^{a}$ D. Astolfi is with Université Claude Bernard Lyon 1, CNRS, LAGEPP UMR 5007, 43 Boulevard du 11 Novembre 1918, F-69100, Villeurbanne, France (daniele.astolfi@univ-lyonl.fr).

${ }^{b}$ Postoyan is with the Universite de Lorraine, CNRS, CRAN, F-54000 Nancy, France. (romain.postoyan@univ-lorraine.fr).

${ }^{c}$ D. Nešić is with the Department of Electrical and Electronic Engineering, The University of Melbourne, Parkville, 3010 Victoria Australia. (dnesic@unimelb.edu.au).

The first author was partially financially supported by the "Région GrandEst" of France.

${ }^{1}$ Fundamental limitations arise, see [1] in the context of linear systems. approach, often used by practitioners, have been studied by means of switching, adaptive or gain-scheduling strategies, see for instance [6]-[10].

The main limitation of these works is that these apply to specific classes of systems or observers. To the best of our knowledge, if we are given two observers, each of them satisfying a subset of properties (a)-(d), no general methodology is available to unite them in a single estimation scheme satisfying all the properties (a)-(d). Such results exist, on the other hand, in the context of control, see e.g., [11]-[14].

The objective of this paper is therefore to propose a framework to combine two given observers in order to obtain a uniting observer inheriting the "good features" of each of them, thus providing a rigorous foundation and guidelines for this technique, which is commonly used in practice. We provide a set of assumptions on the observers and we then explain how to unite them using a hybrid scheme. The two initial observers can be constructed using various techniques borrowed from the literature, see e.g., [2], [4], [7], [15]-[27], and they do not need to be of the same type and of the same dimension, namely we can unite a HGO and an EKF for instance.

Inspired by [12], where the problem of uniting two outputfeedback controllers is addressed, we suppose to know two observers. One is denoted as the global observer and ensures a global ${ }^{2}$ domain of attraction, and possibly a certain rate of convergence. The other one is referred to as the local observer, its convergence is only ensured in a neighborhood of the plant trajectory and guarantees some desired performances in presence of measurement noise. The idea we pursue is to use the former when the estimation error is large, namely during transients, and latter when the estimation error is small, namely the asymptotic behaviour. A hysteresis switching mechanism is used to select one observer at a time. This mechanism, in the ideal case, should be based on the state estimation errors produced by the two observers. However, the state estimation errors are not directly available. As a consequence, these are estimated by means of a dynamical system processing the output of the plant and the outputs of the observers. The construction follows the design of norm estimators proposed in [28] for input-output-to-state stable (IOSS) systems. The proposed switching mechanism is able to switch back and forth between the two observers. At the end, the resulting uniting observer guarantees the following properties: global convergence of the estimation error; finite number of switches under some conditions on the measurement noise; asymptotic behavior coinciding with that of the local observer.

\footnotetext{
${ }^{2}$ The notion of global refers, in this work, to a given (arbitrarily large) set of initial conditions and not necessarily with respect to the full state space.
} 
Two case studies are presented to illustrate the applicability of the framework. We first unite an EKF with a globally asymptotically convergent observer, which can be designed by applying any of the techniques in [2], [15], [17], [20]-[26], [29]. The interest here is to exploit the local good properties of the EKF together with the global convergence of the other observer. We then study the scenario where the same type of global observer is available, except that the convergence is no longer asymptotic but approximate, in the sense that the estimation error does not converge to zero with time, but "close" to it. This case is interesting when we do not know a global asymptotic observer, but we have an EKF, which we would like to work globally.

The main ideas of this work are inspired by the dual problem of uniting controllers addressed in [12]. Although the proposed design is conceptually similar to [12], the problems of uniting controllers and uniting observers are very different from the technical point of view. The main difference is that, in the former, one has to know how far is the current system state from the desired equilibrium point, while in the latter, we need to know the distance between the observer estimate and the current system state, which is in general a time-varying trajectory, and thus more challenging. Although in the case of linear systems these two problems coincide, this is no longer true in presence of nonlinear dynamics and results to be a crucial difference between the stabilization of an equilibrium and the stabilization of a time-varying trajectory. This issue arises in a large number of control problems, such as observer design, tracking, output regulation, or synchronization. For this reason, it is not possible to use "off-the shelf" the results proposed in [12] in the context of stabilization to solve the problem of uniting observers: different assumptions, design and analysis tools are needed.

Compared to the preliminary version of this work in [30], completely novel elements include: a new design based on different assumptions; the explicit consideration of the measurement noise; two case studies. Finally, the construction proposed in [30] is presented in Section III-D with its complete proof, contrary to [30].

The rest of the paper is organized as follows. Notations and definitions are briefly recalled in Section II. We provide the main result in Section III. Then, the two case studies are presented in Section IV. A numerical example is given in Section V. The proofs of the results are detailed in Section VI. Finally, conclusions and future perspectives are discussed in Section VII.

\section{PRELIMINARIES}

The notation $\mathbb{R}$ stands for the set of real numbers, $\mathbb{R}_{\geq 0}:=$ $[0, \infty), \mathbb{Z}$ is the set of integer numbers, $\mathbb{Z}_{\geq 0}:=\{0,1,2, \ldots\}$ and $\mathbb{Z}_{>0}:=\{1,2, \ldots\}$. Given $x \in \mathbb{R}^{n}$ and $y \in \mathbb{R}^{m}$, we denote $(x, y):=\left(x^{\top}, y^{\top}\right)^{\top}$. The notation $|x|$, with $x \in \mathbb{R}^{n}$, stands for the standard Euclidean norm, while $|A|$, with $A \in \mathbb{R}^{m \times n}$ is used for the standard induced matrix norm. A continuous function $\alpha: \mathbb{R}_{\geq 0} \rightarrow \mathbb{R}_{\geq 0}$ is of class $\mathcal{K}$ if it is strictly increasing and $\alpha(0)=0$, and it is of class $\mathcal{K}_{\infty}$ if, in addition, $\lim _{r \rightarrow \infty} \alpha(r)=\infty$. A continuous function $\beta: \mathbb{R}_{\geq 0} \times \mathbb{R}_{\geq 0} \rightarrow$
$\mathbb{R}_{\geq 0}$ is of class $\mathcal{K} \mathcal{L}$ if, for any $s \in \mathbb{R}_{\geq 0}, \beta(\cdot, s) \in \mathcal{K}$, and, for each $r \in \mathbb{R}_{\geq 0}$, the function $\beta(r, s)$ is decreasing with $s$ and satisfies $\lim _{s \rightarrow \infty} \beta(r, s)=0$. We compactly write $\alpha \in \mathcal{K}, \alpha \in \mathcal{K}_{\infty}, \beta \in \mathcal{K} \mathcal{L}$. Given a matrix $P \in \mathbb{R}^{n \times m}$, we denote by $\operatorname{vec}(P)$ the vectorization of the matrix, namely $\operatorname{vec}(P):=\left(p_{11}, \ldots, p_{1 m}, p_{21}, \ldots, p_{2 m}, \ldots, p_{n 1}, \ldots, p_{n m}\right)$, where $p_{i j}$ is the $i, j$ entry of $P$. We denote by $\operatorname{diag}\left(a_{1}, \ldots, a_{n}\right)$ a matrix whose diagonal entries are given respectively by $a_{1}, \ldots, a_{n}$ and all the other entries are zero. The symbol $I$ denotes the identity matrix of appropriate dimension which will be made clear by the context.

We consider hybrid systems with state $x \in \mathcal{X} \subseteq \mathbb{R}^{n_{x}}$ and input $u \in \mathcal{U} \subseteq \mathbb{R}^{n_{u}}$ in the formalism of [31], [32], namely

$$
\mathcal{H}:\left\{\begin{aligned}
\dot{x} & =F(x, u), & & (x, u) \in \mathcal{C} \times \mathcal{U}, \\
x^{+} & =G(x, u), & & (x, u) \in \mathcal{D} \times \mathcal{U},
\end{aligned}\right.
$$

where $\mathcal{C} \subseteq \mathcal{X}$ is the flow set, $\mathcal{D} \subseteq \mathcal{X}$ is the jump set, $F$ is the flow map and $G$ is the jump map. Solutions to system (1) are defined on hybrid time domains. A set $E \subset \mathbb{R}_{>0} \times \mathbb{Z}_{>0}$ is a compact hybrid time domain if $E=\bigcup_{j=0}^{J}\left(\left[t_{j}, t_{j+1}\right], j\right)$ for some finite sequence of times $0=t_{0} \leq t_{1} \leq \ldots \leq t_{J+1}$ and it is a hybrid time domain if for all $(T, J) \in E, E \cap([0, T] \times\{0,1, \ldots, J\})$ is a compact hybrid time domain. On each hybrid time domain we use the natural ordering relation $\left(t_{0}, j_{0}\right) \preceq\left(t_{1}, j_{1}\right)$ if $t_{0}+j_{0} \leq t_{1}+j_{1}$. Given a hybrid time domain $E$, we define $\sup _{t} E:=\sup \left\{t \in \mathbb{R}_{\geq 0}: \exists j \in \mathbb{Z}_{\geq 0}\right.$ such that $\left.(t, j) \in E\right\}$, $\sup _{j} E:=\sup \left\{j \in \mathbb{Z}_{\geq 0}: \exists t \in \mathbb{R}_{\geq 0}\right.$ such that $\left.(t, j) \in E\right\}$. A hybrid signal is a function defined on a hybrid time domain. A hybrid signal $u: \operatorname{dom} u \rightarrow \mathcal{U}$ is called a hybrid input if $u(\cdot, j)$ is measurable and locally essentially bounded for each $j$. A hybrid signal $x: \operatorname{dom} x \rightarrow \mathcal{X}$ is called a hybrid arc if $x(\cdot, j)$ is locally absolutely continuous for each $j$. A hybrid $\operatorname{arc} x: \operatorname{dom} x \rightarrow \mathcal{X}$ and a hybrid input $u: \operatorname{dom} u \rightarrow \mathcal{U}$ is a solution pair $(x, u)$ to $\mathcal{H}$ in if $\operatorname{dom} x=\operatorname{dom} u$, $(x(0,0), u(0,0)) \in(\mathcal{C} \cup \mathcal{D}) \times \mathcal{U}$, and

- for all $j \in \mathbb{Z}_{\geq 0}$ and almost all $t$ such that $(t, j) \in \operatorname{dom} x$, $(x(t, j), u(t, \bar{j})) \in \mathcal{C} \times \mathcal{U}$ and $\dot{x}=F(x(t, j), u(t, j))$;

- for all $(t, j) \in \operatorname{dom} x$ such that $(t, j+1) \in$ $\operatorname{dom} x,(x(t, j), u(t, j)) \in \mathcal{D} \times \mathcal{U}$ and $x(t, j+1)=$ $G(x(t, j), u(t, j))$.

A solution pair $(x, u)$ to $\mathcal{H}$ is maximal if it cannot be extended and it is complete if $\operatorname{dom} x$ is unbounded. In the sequel, each time we talk of solutions we mean maximal solutions.

Given any hybrid signal $w: \operatorname{dom} w \rightarrow \mathbb{R}^{n_{w}}$, we define $\|w\|_{\infty}:=\max \left\{\sup _{(t, j) \in \Gamma(w)}|w(t, j)|\right\}$ where $\Gamma(w)$ denotes the set of all $(t, j) \in \operatorname{dom} w$ such that $(t, j+1) \in \operatorname{dom} w$.

By adopting the same notation, we denote by $(x, u)$ a solution pair, with the $x$-component initialized in $\mathcal{X}$ and control $u$ taking values in $\mathcal{U}$, to a differential equation of the form $\dot{x}=f(x, u)$, with state $x \in \mathcal{X} \subseteq \mathbb{R}^{n_{x}}$ and input $u \in \mathcal{U} \subseteq \mathbb{R}^{n_{u}}$. Given any continuous signal $w:[0, \infty) \rightarrow \mathbb{R}^{n_{w}}$, we define $\|w\|_{\infty}:=\sup _{t \in[0, \infty)}|w(t)|$.

\section{UNiting OBSERVER DESIGN}

This section is structured as follows. We formulate the uniting observer problem in Section III-A. Then, we present 
the assumptions we make in Section III-B. The main result, in which we show how to design a hybrid observer solving the uniting observer problem, is given in Section III-C. An alternative design, based on different assumptions, is finally proposed in Section III-D.

\section{A. Problem Statement}

We consider nonlinear systems of the form

$$
\dot{x}=f(x, u), \quad y=h(x, w),
$$

where $x \in \mathcal{X} \subseteq \mathbb{R}^{n_{x}}$ is the state, $u \in \mathcal{U} \subseteq \mathbb{R}^{n_{u}}$ is a known input, $y \in \mathbb{R}^{n_{y}}$ is the measured output, and $w \in \mathcal{W} \subseteq \mathbb{R}^{n_{w}}$ represents an unknown measurement noise, with $n_{x}, n_{u}, n_{y}, n_{w} \in \mathbb{Z}_{>0}$. The sets $\mathcal{X}, \mathcal{U}, \mathcal{W}$ are closed, the functions $f, h$ are locally Lipschitz, and the signals corresponding to $u$ and $w$ in (1) are defined for all positive times, Lebesgue measurable and locally essentially bounded.

We assume that we know two observers for system (1). One is referred to as the local observer, and the other one as the global observer. The local observer is the one we want to use when the estimation error is small, while the global observer guarantees that the estimation error becomes eventually sufficiently small for any possible initialization. The state variables and the functions related to those observers will be indexed respectively with 0 (local) and 1 (global).

The dynamics of the local observer is described by

$$
\dot{\zeta}_{0}=\varphi_{0}\left(\zeta_{0}, u, y\right), \quad \hat{x}_{0}=\vartheta_{0}\left(\zeta_{0}\right), \quad \hat{y}_{0}=h\left(\hat{x}_{0}, 0\right),
$$

where $\zeta_{0} \in \mathcal{Z}_{0} \subseteq \mathbb{R}^{n_{0}}$ is the observer state, $n_{0} \in \mathbb{Z}_{>0}$ is the observer dimension satisfying ${ }^{3} n_{0} \geq n_{x}$, and $\hat{x}_{0} \in \mathbb{R}^{n_{x}}$ is the estimate of $x$. The set $\mathcal{Z}_{0}$ is closed and the functions $\varphi_{0}, \vartheta_{0}$ are assumed to be locally Lipschitz. Loosely speaking, observer (2) is local and asymptotic in the sense that if the initial estimation error $\left|x(0)-\hat{x}_{0}(0)\right|$ is small enough, then convergence of the estimation error in absence of measurement noise is guaranteed, namely $\lim _{t \rightarrow \infty}|x(t)-\hat{x}(t)|=0$. This property is rigorously stated in Section III-B. Although any observer which has global (or semiglobal) convergence properties satisfy this condition, local observers are of particular interest because they are often easy to design and they usually possess good robustness properties in presence of (small) measurement noise. A typical example is the EKF or its variations, see e.g., [4], [5], [16], [19].

The global observer for system (1) is of the form

$$
\dot{\zeta}_{1}=\varphi_{1}\left(\zeta_{1}, u, y\right), \quad \hat{x}_{1}=\vartheta_{1}\left(\zeta_{1}\right), \quad \hat{y}_{1}=h\left(\hat{x}_{1}, 0\right),
$$

where $\zeta_{1} \in \mathcal{Z}_{1} \subseteq \mathbb{R}^{n_{1}}$ is the observer state, $n_{1} \in \mathbb{Z}_{>0}$ is the observer dimension satisfying $n_{1} \geq n_{x}$ ( similarly to (2)), and $\hat{x}_{1} \in \mathbb{R}^{n_{x}}$ is the estimate of $x$. The set $\mathcal{Z}_{1}$ is closed and the functions $\varphi_{1}, \vartheta_{1}$ are assumed to be locally Lipschitz. Examples of global observers can be found in [2], [15], [22], [26], [33] for $n_{1}=n_{x}$ and in [21], [23]-[25], [27], [34] for $n_{1}>n_{x}$. Other examples are given in Section IV.

The main idea of this work is to combine observers (2) and (3) in order to benefit from the advantages of each of them. To

\footnotetext{
${ }^{3}$ We do not consider reduced order observers, namely observers with $n_{0}<$ $n_{x}$, though all the forthcoming results can be adapted to cover this case.
}

address this problem, we aim at designing a hybrid observer of the general form below, based on (2) and (3),

$$
\begin{aligned}
\dot{\xi} & =F(\xi, u, y), \quad \xi \in \mathcal{C}, \\
\xi^{+} & =G(\xi, u, y), \quad \xi \in \mathcal{D}, \\
\hat{x} & =H(\xi),
\end{aligned}
$$

where $\xi \in \mathbb{R}^{n_{\xi}}$ is the observer state, $n_{\xi} \in \mathbb{Z}_{>0}, \mathcal{C} \subseteq \mathbb{R}^{n_{\xi}}$ and $\mathcal{D} \subseteq \mathbb{R}^{n_{\xi}}$ are closed set, and $\hat{x}$ is the estimate of $x$. As a result, system (1) and observer (4) lead to the overall hybrid system below

$$
\left.\begin{array}{rl}
\dot{x} & =f(x, u) \\
\dot{\xi} & =F(\xi, u, y)
\end{array}\right\} \quad(x, \xi, u, w) \in \mathcal{X} \times \mathcal{C} \times \mathcal{U} \times \mathcal{W},
$$

Note that $(u, w)$ needs to be defined on hybrid time domains in (5). With some abuse of notation, we consider, throughout the rest of the paper, $u$ and $w$, that are defined in such way that their values agree with $(u(t), w(t))$ during flows, do not change during jumps, and their hybrid time domains correspond to that of $(x, \xi)$.

Our objective is to construct (4) to solve the problem stated next.

Definition 1 (Uniting observer). The observer (4) solves the uniting problem for system (1) if the following holds.

(a) (Completeness of solutions and finite number of jumps) Any solution pair ${ }^{4}((x, \xi),(u, w))$ to (5) is complete and satisfies $\sup _{t} \operatorname{dom}(x, \xi)=+\infty, \sup _{j} \operatorname{dom}(x, \xi)<\infty$.

(b) (Global convergence) There exists $\gamma \in \mathcal{K}$ such that any solution pair $((x, \xi),(u, w))$ to $(5)$ satisfies

$$
\limsup _{t+j \rightarrow \infty}|x(t, j)-\hat{x}(t, j)| \leq \gamma\left(\|w\|_{\infty}\right)
$$

(c) (Local behaviour) There exists a set $\mathcal{B} \subseteq \mathcal{X} \times(\mathcal{C} \cup \mathcal{D})$ such that any solution pair $((x, \xi),(u, w))$ to (5) with $(x(0,0), \xi(0,0)) \in \mathcal{B}$, has hybrid time domain $[0, \infty) \times$ $\{0\}$, and $\hat{x}(t, 0)=\hat{x}_{0}(t)$ for all $t \in[0, \infty)$, where $\hat{x}_{0}$ is a solution to (1), (2).

Item (a) of Definition 1 means that the solutions of the hybrid observer (4) are complete, that no Zeno behaviour can occur and that switches stop in finite time. Item (b) of Definition 1 ensures that the estimation error has an asymptotic gain property with respect to the measurement noise with a global domain of attraction ${ }^{5}$. When there is no noise, i.e. $w=0$, the global asymptotic convergence of the estimation error $x-\hat{x}$ is ensured. Finally, item (c) of Definition 1 guarantees that only the local observer (2) is used if observer (4) is initialized in such way that $(x, \xi)$ is in the set $\mathcal{B}$ at the initial time.

\footnotetext{
${ }^{4}$ Recall that each time we mention a solution, it is a maximal one, see the end of Section II.

${ }^{5}$ Global with respect to the domain of definition of system (5), that is $\mathcal{X} \times(\mathcal{C} \cup \mathcal{D})$.
} 


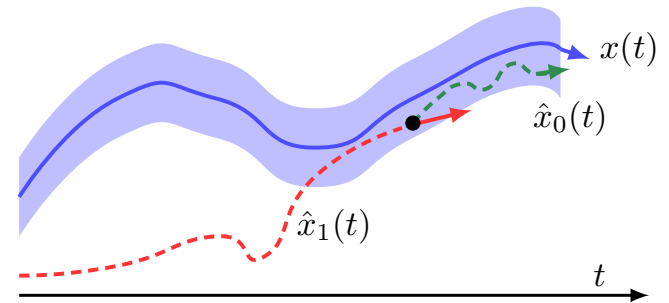

Fig. 1: Strategy to unite local and global observers: when the estimate is far from the current state we use the global observer; then, when the estimate of the global observer is close enough to the actual trajectory of the plant, we activate the local observer initialized at the estimate given by the global observer. Blue line: trajectory $x(t)$ of system (1). Dotted red line: trajectory $\hat{x}_{1}(t)$ of global observer (3). Dotted green line: trajectory $\hat{x}_{0}(t)$ of local observer (2).

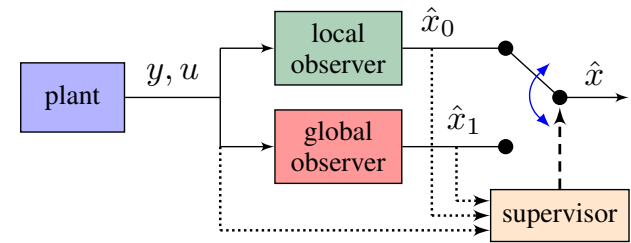

Fig. 2: Hybrid scheme to unite local observer (2) and global observer (3).

The rationale of the scheme we construct in the following is illustrated in Figure 1. We want to use the global observer (3) during transients, when the estimation error is large. Then, when $\left|\hat{x}_{1}-x\right|$ is small enough, we reset the variables of the local observer (2) according to the estimate $\hat{x}_{1}$ provided by the global observer. Afterwards, we let the local observer run. This switching mechanism cannot be implemented in open loop, i.e. based on time only, for robustness reasons. We want a supervisor mechanism, which is able to switch between the two observers in order to cope with possible wrong initializations or large disturbances $w$, as depicted in Figure 2. This mechanism therefore needs to rely on $\left|\hat{x}_{0}-x\right|$ and $\left|\hat{x}_{1}-x\right|$, but these quantities are not accessible since we do not know $x$. Inspired by [12], [28], we use instead estimates of these values based on the measured output $y$ and the estimated outputs $\hat{y}_{0}, \hat{y}_{1}$. To do so, we need to make several assumptions, which are now presented.

\section{B. Assumptions}

First of all, we suppose that the dynamics of system (1) and observers (2) and (3) are well posed in the sense that solutions are defined for all $t \geq 0$.

Standing Assumption (System and observers dynamics). The following holds.

(i) For any initial condition $\left(x(0), \zeta_{0}(0), \zeta_{1}(0)\right) \in \mathcal{X} \times \mathcal{Z}_{0} \times$ $\mathcal{Z}_{1}$, and any input $(u, w)$ taking values in $\mathcal{U} \times \mathcal{W}$, any corresponding solution pair to (1), (2), (3) is unique, defined on $[0, \infty)$, and $\left(x(t), \zeta_{0}(t), \zeta_{1}(t)\right) \in \mathcal{X} \times \mathcal{Z}_{0} \times \mathcal{Z}_{1}$ for all $t \in[0, \infty)$. (ii) There exists a function $\Theta: \mathbb{R}^{n_{1}} \rightarrow \mathbb{R}^{n_{0}}$ such that $\Theta\left(\zeta_{1}\right) \in$ $\mathcal{Z}_{0}$ and $\vartheta_{1}\left(\zeta_{1}\right)=\vartheta_{0}\left(\Theta\left(\zeta_{1}\right)\right)$ for all $\zeta_{1} \in \mathcal{Z}_{1}$, where $\vartheta_{0}, \vartheta_{1}$ come from (2) and (3), respectively.

Item (i) of Standing Assumption states that the trajectories of the system (1) and of the observers (2), (3) are well defined and lie in the sets $\mathcal{X}, \mathcal{Z}_{0}, \mathcal{Z}_{1}$ for all times. As a consequence, these sets will in general be chosen large. In some cases, the dynamics of the observers may need to be modified to guarantee this property. This can be done for instance by using the technique proposed in [27] or by modifying the definition of the jump maps and the flow and jump sets in (4), by resetting the state $\zeta_{0}$ inside $\mathcal{Z}_{0}$ when it is close to the boundary of $\mathcal{Z}_{0}, \mathcal{Z}_{1}$. We do not address this last issue in order not to blur our the main message of this work.

Function $\Theta$ in item (ii) of Standing Assumption is needed to map the global observer variable $\zeta_{1}$ to the local observer variable $\zeta_{0}$, which is essential when the local observer is activated. In particular, when this occurs, we will take $\zeta_{0}^{+}=\Theta\left(\zeta_{1}\right)$. Hence, since $\Theta\left(\zeta_{1}\right) \in \mathcal{Z}_{0}$ for any $\zeta_{1} \in \mathcal{Z}_{1}$, we are sure that the reset value of $\zeta_{0}$ lies in $\mathcal{Z}_{0}$, as required. Moreover, item (ii) ensures also that $\hat{x}_{0}^{+}=\hat{x}_{1}$. When the dimensions of the observers coincide and their dynamics are expressed in the same coordinates, $\Theta$ is simply the identity map. Additional examples of $\Theta$ are provided in Section IV.

The next assumption formalizes the properties of local observer (2).

Assumption 1 (Local observer). There exist a continuous function $V_{0}: \mathcal{X} \times \mathcal{Z}_{0} \rightarrow \mathbb{R}_{\geq 0}, \gamma_{0} \in \mathcal{K}$, and $\varepsilon_{0}^{\prime}>\varepsilon_{0}>0$, such that the following holds.

(i) (Local convergence) Any solution pair $\left(\left(x, \zeta_{0}\right),(u, w)\right)$ to (1), (2), with initial condition such that $V_{0}\left(x(0), \zeta_{0}(0)\right) \leq$ $\varepsilon_{0}^{\prime}$, satisfies $\lim \sup _{t \rightarrow \infty}\left|x(t)-\hat{x}_{0}(t)\right| \leq \gamma_{0}\left(\|w\|_{\infty}\right)$.

(ii) (Invariance property) Any solution pair $\left(\left(x, \zeta_{0}\right),(u, w)\right)$ to (1), (2), with initial condition such that $V_{0}\left(x(0), \zeta_{0}(0)\right) \leq$ $\varepsilon_{0}$, satisfies $V_{0}\left(x(t), \zeta_{0}(t)\right) \leq \varepsilon_{0}$ for all $t \in[0, \infty)$.

The function $V_{0}$ is used to characterize the domain of attraction of local observer (2), which contains the set $\left\{\left(x, \zeta_{0}\right): V_{0}\left(x, \zeta_{0}\right) \leq \varepsilon_{0}^{\prime}\right\}$, see item (i) of Assumption 1 . On the other hand, when the initial estimation error satisfies $V_{0}\left(x(0), \zeta_{0}(0)\right) \leq \varepsilon_{0}^{\prime}$, then it has an asymptotic gain property with respect to the measurement noise $w$, and converges asymptotically to zero when $w(t)=0$ for all $t \geq 0$, since $\gamma_{0}(0)=0$, according to item (i) of Assumption 1. Function $V_{0}$ typically corresponds to the Lyapunov function that is used to prove the convergence of the local observer and satisfies $\underline{\alpha}_{0}\left(\left|x-\hat{x}_{0}\right|\right) \leq V\left(x, \zeta_{0}\right)$ for some $\underline{\alpha}_{0} \in \mathcal{K}$. According to this interpretation, $\left\{\left(x, \zeta_{0}\right): V_{0}\left(x, \zeta_{0}\right) \leq \varepsilon_{0}\right\}$ can be viewed as an invariant Lyapunov level set of size $\varepsilon_{0}$, see item (ii) of Assumption 1. In general the values of $\varepsilon_{0}^{\prime}, \varepsilon_{0}$ depend on the maximum allowed magnitude of the measurement noise, namely on $\sup _{w \in \mathcal{W}}|w|$.

In the next assumption we suppose that function $V_{0}$, evaluated along the solutions to (1), (2), can be overestimated by a dynamical system. The latter will be essential to detect when to activate the local observer. 
Assumption 2 (Estimator of $V_{0}$ ). There exist a continuous function $\rho_{0}: \mathbb{R}^{n_{y}} \times \mathbb{R}^{n_{y}} \rightarrow \mathbb{R}_{>0}$ satisfying $\rho_{0}(y, y)=0$ for any $y \in \mathbb{R}^{n_{y}}, \beta_{0} \in \mathcal{K} \mathcal{L}, a_{0}, b_{0}, c_{0}>0$ and $v_{0} \geq 0$, such that, under Assumption 1, the following holds.

(i) Any solution pair $\left(\left(x, \zeta_{0}\right),(u, w)\right)$ to (1), (2), satisfies

$V_{0}\left(x(t), \zeta_{0}(t)\right) \leq a_{0} z_{0}(t)+\beta_{0}\left(V_{0}\left(x(0), \zeta_{0}(0)\right)+z_{0}(0), t\right)+v_{0}$

for all $t \geq 0$, where $z_{0}$ is the solution to

$$
\dot{z}_{0}=-b_{0} z_{0}+\rho_{0}\left(y, \hat{y}_{0}\right)
$$

with initial condition $z_{0}(0) \in \mathbb{R}_{\geq 0}$.

(ii) The function $\rho_{0}$ satisfies

$\sup \left\{\rho_{0}\left(y, \hat{y}_{0}\right):\left(x, \zeta_{0}\right) \in \mathcal{X} \times \mathcal{Z}_{0}, V_{0}\left(x, \zeta_{0}\right) \leq \varepsilon_{0}\right.$

where $y=h(x, w)$ and $\hat{y}_{0}=h\left(\vartheta_{0}\left(\zeta_{0}\right), 0\right)$. $w \in \mathcal{W}\} \leq b_{0} c_{0}$

(iii) $\varepsilon_{0} \leq a_{0} c_{0}<\varepsilon_{0}^{\prime}-v_{0}$.

Assumption 2 states that function $V_{0}$ can be overestimated via the dynamical system (7), which is called in the following as a norm estimator, to be consistent with the terminology coined in [28]. In particular, in view of item (i) of Assumption 2 , and the fact that $\beta_{0} \in \mathcal{K} \mathcal{L}$, the state $z_{0}$ asymptotically provides an upper bound of $V_{0}$, up to the constant $v_{0}$. The norm estimator (7) can thus be used to detect whether the state of the local observer (2) is in the domain of attraction $V_{0}\left(x, \zeta_{0}\right) \leq \varepsilon_{0}^{\prime}$, established by Assumption 1. For this, note that if $\left(x(t), \zeta_{0}(t)\right)$ satisfies $V_{0}\left(x(t), \zeta_{0}(t)\right) \leq \varepsilon_{0}$ for all $t \geq 0$, then, in view of (7) and item (ii) of Assumption 2, we obtain $\lim _{t \rightarrow \infty} z_{0}(t) \leq c_{0}$, which implies, in view of items (i) and (iii) of Assumption 2 and the properties of $\beta_{0}$, that $\limsup _{t \rightarrow \infty} V_{0}\left(x(t), \zeta_{0}(t)\right) \leq a_{0} c_{0}+v_{0}<\varepsilon_{0}^{\prime}$. In other words, any solution $\left(x, \zeta_{0}\right)$ satisfying $z_{0} \leq c_{0}$ for a large enough amount of time ensures that the local observer is asymptotically converging to the plant state, up to the perturbing term due to $w$, see item (i) of Assumption 1.

Item (i) of Assumption 2 is always satisfied when the system is uniformly observable (see [29]), namely when a global asymptotic observer exists, as shown in [7] and explained in Section IV. If there exist $\varrho_{0}, \psi_{0} \in \mathcal{K}$ such that the function $V_{0}$ satisfies, along any solution to (1), (2), the following differential inequality

$$
\dot{V}_{0} \leq-b_{0} V_{0}+\varrho_{0}\left(\left|y-\hat{y}_{0}\right|\right)+\psi_{0}(|w|),
$$

then, item (i) of Assumption 2 is verified by selecting any $\rho_{0}$ such that $\rho_{0}\left(s_{1}, s_{2}\right) \geq \varrho_{0}\left(\left|s_{1}-s_{2}\right|\right)$ for all $s_{1}, s_{2} \in \mathbb{R}^{n_{y}}$ and $v_{0}=b_{0}^{-1} \sup _{w \in \mathcal{W}} \psi_{0}(|w|)$. To see this, it suffices to apply the comparison principle to the following differential inequality obtained by subtracting (7) to (8)

$$
\begin{aligned}
\dot{V}_{0}-\dot{z} & \leq-b_{0}\left(V_{0}-z_{0}\right)+\varrho_{0}\left(\left|y-\hat{y}_{0}\right|\right)-\rho_{0}\left(y, \hat{y}_{0}\right)+\psi_{0}(|w|) \\
& \leq-b_{0}\left(V_{0}-z_{0}\right)+b_{0} v_{0} .
\end{aligned}
$$

Item (ii) of Assumption 2 is then satisfied by properly selecting the constant $a_{0}, c_{0}$. Note that the constant $v_{0} \geq 0$ is a bias introduced by the measurement noise. When the noise is not present, we have in general $v_{0}=0$; however, if it is too large, item (iii) of Assumption 2 may not be satisfied. This means that our results are, in general, valid for "small" noise.
Depending on the design of the observer, the function $\varrho_{0}$ in (8) may be a degree of freedom or imposed by the structure of $V_{0}$. See further examples in Section IV.

In the next assumption, we define the properties of the global observer (3).

Assumption 3 (Global observer). There exist a continuous function $V_{1}: \mathcal{X} \times \mathcal{Z}_{1} \rightarrow \mathbb{R}_{\geq 0}$ and $\varepsilon_{1}>0$ such that, any solution pair $\left(\left(x, \zeta_{1}\right),(u, w)\right)$ to (1), (3), satisfies $\lim \sup _{t \rightarrow \infty} V_{1}\left(x(t), \zeta_{1}(t)\right)<\varepsilon_{1}$.

The function $V_{1}$ is used to characterize the asymptotic behaviour of the global observer. Typically, the function $V_{1}$ is the Lyapunov function constructed to show the convergence of the global observer and satisfies $\underline{\alpha}_{1}\left(\left|x-\hat{x}_{1}\right|\right) \leq V_{1}\left(x, \zeta_{1}\right)$ for some $\underline{\alpha}_{1} \in \mathcal{K}_{\infty}$. In this case, $\varepsilon_{1}$ characterizes the ultimate bound of the estimation error of (3) and depends, in general on the magnitude of the measurement noise, namely on $\sup _{w \in W}|w|$. When $w=0$, the value of $\varepsilon_{1}$ depends on the properties of the observer (3) and may be selected arbitrarily small if the observer is asymptotically convergent.

The next assumption states that function $V_{1}$ can be overestimated by a dynamical system.

Assumption 4 (Estimator of $V_{1}$ ). There exist a continuous function $\rho_{1}: \mathbb{R}^{n_{y}} \times \mathbb{R}^{n_{y}} \rightarrow \mathbb{R}_{\geq 0}, a_{1}, b_{1}, c_{1}, \varepsilon_{1}^{\prime}>0$, and $v_{1} \geq 0$, such that, under Assumption 3, the following holds.

(i) Any solution pair $\left(\left(x, \zeta_{1}\right),(u, w)\right)$ to (1), (3), satisfies

$V_{1}\left(x(t), \zeta_{1}(t)\right) \leq a_{1} z_{1}(t)+\beta_{1}\left(V_{1}\left(x(0), \zeta_{1}(0)\right)+z_{1}(0), t\right)+v_{1}$

for all $t \in[0, \infty)$, where $z_{1}$ is the solution to

$$
\dot{z}_{1}=-b_{1} z_{1}+\rho_{1}\left(y, \hat{y}_{1}\right)
$$

with initial condition $z_{1}(0) \in \mathbb{R}_{\geq 0}$.

(ii) The function $\rho_{1}$ satisfies

$\sup \left\{\rho_{1}\left(y, \hat{y}_{1}\right):\left(x, \zeta_{1}\right) \in \mathcal{X} \times \mathcal{Z}_{1}, V_{1}\left(x, \zeta_{1}\right) \leq \varepsilon_{1}\right.$,

where $y=h(x, w)$ and $\hat{y}_{1}=h\left(\vartheta_{1}\left(\zeta_{1}\right), 0\right)$. $w \in \mathcal{W}\} \leq b_{1} c_{1}$, (iii) $\varepsilon_{1} \leq a_{1} c_{1}<\varepsilon_{1}^{\prime}-v_{1}$.

Assumption 4 is the counterpart of Assumption 2 for global observer (3) and the same interpretation holds for the function $V_{1}$ and its norm estimator (9).

Finally, for the approach to work, we need the ultimate bound of the estimation error provided by global observer (3) to be included in the basin of attraction of local observer (2). This condition is referred to as the matching condition ${ }^{6}$.

Assumption 5 (Matching condition). For any $\left(x, \zeta_{1}\right) \in$ $\mathcal{X} \times \mathcal{Z}_{1}$ satisfying $V_{1}\left(x, \zeta_{1}\right) \leq \varepsilon_{1}^{\prime}$, then $V_{0}\left(x, \Theta\left(\zeta_{1}\right)\right) \leq \varepsilon_{0}$, where $\Theta$ is defined in the Standing Assumption, $V_{0}, \varepsilon_{0}$ in Assumption 1, $V_{1}$ in Assumption 3 and $\varepsilon_{1}^{\prime}$ in Assumption 4.

Assumption 5 requires that $\left\{\left(x, \zeta_{1}\right): V_{1}\left(x, \zeta_{1}\right) \leq \varepsilon_{1}^{\prime}\right\}$ is included in $\left\{\left(x, \zeta_{0}\right): V_{0}\left(x, \zeta_{0}\right) \leq \varepsilon_{0}\right\}$, when $\zeta_{0}=\Theta\left(\zeta_{1}\right)$, as depicted in Figure 3. Recall that, in view of Assumption 3, solutions to (1), (3) enters in the set $\left\{\left(x, \zeta_{1}\right): V_{1}\left(x, \zeta_{1}\right) \leq \varepsilon_{1}^{\prime}\right\}$. Moreover, this can be detected via the norm estimator (9), see

\footnotetext{
${ }^{6}$ This has not to be confused with the notion of matching condition often used in control theory.
} 


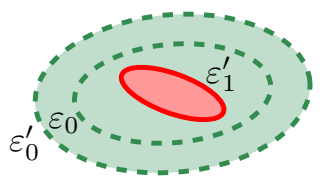

Fig. 3: Matching condition of Assumption 5. Dashed green line: level sets of $V_{0}\left(x, \zeta_{0}\right)$. Red line: level sets of $V_{1}\left(x, \zeta_{1}\right)$.

Assumption 4. As a consequence, Assumption 5 guarantees that, after a sufficient long amount of time, we can reset $\zeta_{0}^{+}=$ $\Theta\left(\zeta_{1}\right)$, with $\Theta$ defined in the Standing Assumption, in order to guarantee asymptotic convergence of the local observer (2), see item (i) of Assumption 1.

Remark 1 (Relaxing the matching condition). Assumption 5 may be difficult to verify. This may occur when the basin of attraction of local observer (2) is too small, namely $\varepsilon_{0}$ is too small; the global observer (3) is not enough accurate, namely $\varepsilon_{1}$ is too large, or the effect of the measurement noise $w$ is too large, namely the level set $\varepsilon_{1}^{\prime}$ of $V_{1}$ that can be estimated via $z_{1}$ is too large. In these cases, one can overcome this problem by enlarging the domain of attraction of the local observer by replacing local observer (2) with a bank of local observers and by using the multi-observer design technique in [10].

\section{Main result}

We are now in the position to state the main result of this paper, namely the design of hybrid observer (4), which solves the uniting observer problem of Definition 1.

The proposed hybrid observer consists of six components: local observer (2), global observer (3), norm estimators (7), (9), a temporal regularization $\tau$, which may be added to prevent undesired consecutive jumps, and a logic variable $q$ taking values in $\{0,1\}$ defining which state estimate, $\hat{x}_{0}$ or $\hat{x}_{1}$, we need to use. In particular, we design the following hybrid observer

$$
\left.\begin{array}{rl}
\dot{\zeta}_{0} & =(1-q) \varphi_{0}\left(\zeta_{0}, u, y\right) \\
\dot{\zeta}_{1} & =\varphi_{1}\left(\zeta_{1}, u, y\right) \\
\dot{z}_{0} & =(1-q)\left(-b_{0} z_{0}+\rho_{0}\left(y, \hat{y}_{0}\right)\right) \\
\dot{z}_{1} & =-b_{1} z_{1}+\rho_{1}\left(y, \hat{y}_{1}\right) \\
\dot{\tau} & =q \\
\dot{q} & =0
\end{array}\right\} \quad \xi \in
$$

in which we use the definitions of $\hat{x}_{0}, \hat{x}_{1}, \hat{y}_{0}$ and $\hat{y}_{1}$, given in (2), (3). The overall state $\xi \in \mathcal{O} \subseteq \mathbb{R}^{n_{\xi}}$, with $\mathcal{O}:=\mathcal{Z}_{0} \times$ $\mathcal{Z}_{1} \times \mathbb{R}^{4}$ and $n_{\xi}:=n_{0}+n_{1}+4$, is therefore defined as

$$
\xi:=\left(\zeta_{0}, \zeta_{1}, z_{0}, z_{1}, \tau, q\right) \in \mathbb{R}^{n_{\xi}}=\mathbb{R}^{n_{0}} \times \mathbb{R}^{n_{1}} \times \mathbb{R}^{4} .
$$

The sets $\mathcal{C} \cup \mathcal{D} \subseteq \mathcal{O}$ are defined as $\mathcal{C}:=\mathcal{C}_{0} \cup \mathcal{C}_{1}$ and $\mathcal{D}:=$ $\mathcal{D}_{0} \cup \mathcal{D}_{1}$, with

$$
\begin{aligned}
& \mathcal{C}_{0}:=\left\{\xi \in \mathcal{O}: z_{0} \in\left[0, c_{0}^{\prime}\right], z_{1} \in[0, \infty), \tau \in[0, \infty),\right. \\
& q=0\}, \\
& \mathcal{C}_{1}:=\left\{\xi \in \mathcal{O}: z_{0}=0,\left(z_{1} \in\left[c_{1}^{\prime}, \infty\right) \text { or } \tau \in[0, T]\right),\right. \\
& q=1\}, \\
& \mathcal{D}_{0}:=\left\{\xi \in \mathcal{O}: z_{0} \in\left[c_{0}^{\prime}, \infty\right), z_{1} \in[0, \infty), \tau \in[0, \infty),\right. \\
& q=0\}, \\
& \mathcal{D}_{1}:=\left\{\xi \in \mathcal{O}: z_{0}=0, z_{1} \in\left[0, c_{1}^{\prime}\right], \tau \in[T, \infty), q=1\right\},
\end{aligned}
$$

where $c_{0}^{\prime}, c_{1}^{\prime}>0$ are design parameters to be properly chosen. According to the definition of the set $\mathcal{C}$, the parameter $T$ is used to enforce a minimum amount of time $T$ of flow after a jump when it is taken strictly positive, when flowing in the set $\mathcal{C}_{1}$ defined in (11b). This is a degree of freedom we allow. Note that according to the definition of (10), we can use the compact notation (4) by defining $F, G, H$ as

$$
\begin{aligned}
& F(\xi, u, y):=\left((1-q) \varphi_{0}\left(\zeta_{0}, u, y\right), \varphi_{1}\left(\zeta_{1}, u, y\right),\right. \\
& (1-q)\left(-b_{0} z_{0}+\rho_{0}\left(y, h\left(\vartheta_{0}\left(\zeta_{0}\right), 0\right)\right)\right. \text {, } \\
& \left.-b_{1} z_{1}+\rho_{1}\left(y, h\left(\vartheta_{1}\left(\zeta_{1}\right), 0\right)\right), q, 0\right) \text {, } \\
& G(\xi, u, y):=\left(q \Theta\left(\zeta_{1}\right)+(1-q) \zeta_{0}, \zeta_{1}, 0, z_{1}, 0,1-q\right), \\
& H(\xi):=(1-q) \vartheta_{0}\left(\zeta_{0}\right)+q \vartheta_{1}\left(\zeta_{1}\right) .
\end{aligned}
$$

The proposed hybrid observer (10) has two different operating modes. When $q=1$, we use global observer (3). Thanks to the norm estimator (9), we can detect when the estimate $\hat{x}_{1}$ is close enough to the true value of the estimated state $x$. The temporal regularization $\tau$ imposes to use global observer for at least a $T$ units of (continuous) time. This is only done to avoid unnecessary multiple consecutive jumps in the scheme (10) and to always enforce a flow after a jump when $T>0$. Note that since $T$ can be chosen arbitrarily small, as we will see, we do not lose in generality in designing the proposed hybrid scheme from a practical point of view. When $q=0$, the estimate is given by the local observer. A wrong behaviour of the local observer, namely when its estimate is not converging to the trajectory of the plant, is detected when the state of norm estimator $z_{0}$ becomes too large. In this case, a jump is imposed and we change the operating mode. Note that, when $q=0$, global observer (3) is still used as a "safeguard". In particular, since the state of global observer (3) is never reset, after a time large enough, we know that its estimate always satisfies the bounds in item (i) of Assumption 4. As a result, unwanted behaviours, such as infinitely many switches in absence of measurement noise, are avoided.

The next theorem states the main result of this paper.

Theorem 1. Suppose Assumptions 1 to 5 hold. Let $T \in[0, \infty)$, $c_{0}^{\prime} \in\left(c_{0}, c_{0}^{\prime \prime}\right)$ with $c_{0}^{\prime \prime}=\frac{\varepsilon_{0}^{\prime}-v_{0}}{a_{0}}$, and $c_{1}^{\prime} \in\left(c_{1}, c_{1}^{\prime \prime}\right)$, with $c_{1}^{\prime \prime}=\frac{\varepsilon_{1}^{\prime}-v_{1}}{a_{1}}$, where $a_{0}, a_{1}, c_{0}, c_{1}, \varepsilon_{0}^{\prime}, \varepsilon_{1}^{\prime}, v_{0}, v_{1}$ are given in Assumptions 1-5. Then the hybrid observer (4), with $\xi, F, G, H, \mathcal{C}, \mathcal{D}$ chosen as in (11), solves the uniting problem

\footnotetext{
${ }^{7}$ This choice is always possible since $a_{0} c_{0}<\varepsilon_{0}^{\prime}-v_{0}$ in view of item (iii) of Assumption 2, and $a_{1} c_{1}<\varepsilon_{1}^{\prime}-v_{1}$ in view of item (iii) of Assumption 4.
} 
with $\gamma=\gamma_{0}$ and $\mathcal{B}:=\left\{(x, \xi) \in \mathcal{X} \times \mathcal{O}: V_{0}\left(x, \zeta_{0}\right) \leq \varepsilon_{0}, z_{0} \in\right.$ $\left.\left[0, c_{0}\right], z_{1} \in[0, \infty), \tau \in[0, \infty), q=0\right\}$, where $\gamma_{0}, V_{0}$ are given by Assumption 1.

Remark 2 (Effect of measurement noise). The conditions of Theorem 1 typically require the noise $w$ to be small, as already mentioned. When a large measurement noise is considered, some of the previous assumptions may no longer hold. However, as long as the behaviour of the global observer is well defined, and finite escape time of the local observer do not occur, the scheme proposed in (11) guarantees completeness of solutions. It may happen, however, that infinitely many switches occur as the local observer fails to converge and the global observer moves persistently back and forth from the set $\left\{\left(x, \zeta_{1}\right): V_{1}\left(x, \zeta_{1}\right) \leq \varepsilon_{1}^{\prime}\right\}$.

\section{Alternative design}

In certain cases, Assumption 2 may not be verified, namely it is not possible to design a norm estimator for the local observer. For instance, this case occurs when item (i) of Assumption 2 does not hold globally but only locally, namely item (i) holds only for solutions to (1), (2), for which the estimation error $x-\hat{x}_{0}$ is small enough. In this case, it may be possible to follow the alternative design proposed in [30]. This approach does not rely on Assumption 2, but compares instead the estimates $\hat{x}_{0}, \hat{x}_{1}$, provided by the local and the global observer, to obtain an overestimate of $V_{0}$. For this to work, we need, in addition to Assumptions 1, 3, 4, and 5, the next conditions to hold.

Assumption 6 (Invariance property of the global observer). Any solution pair $\left(\left(x, \zeta_{1}\right),(u, w)\right)$ to (1), (3), starting in $V_{1}\left(x(0), \zeta_{1}(0)\right) \leq \varepsilon_{1}$, with $\varepsilon_{1}$ given in Assumption 1, satisfies $V_{1}\left(x(t), \zeta_{1}(t)\right) \leq \varepsilon_{1}^{\prime}$ for all $t \geq 0$, with $\varepsilon_{1}^{\prime}$ given in Assumption 5.

Assumption 6 states that the set $\left\{\left(x, \zeta_{1}\right): V_{1}\left(x, \zeta_{1}\right) \leq \varepsilon_{1}^{\prime}\right\}$ is invariant for solutions to (1), (3) initialized in $\left\{\left(x, \zeta_{1}\right)\right.$ : $\left.V_{1}\left(x, \zeta_{1}\right) \leq \varepsilon_{1}\right\}$. This extra condition is needed to guarantee the local behaviour property (c) in Definition 1 of local observer (2).

Next assumption shows how to overestimate the function $V_{0}$ starting from the knowledge of $V_{1}, \hat{x}_{1}$ and $\hat{x}_{0}$.

Assumption 7 (Estimation of $V_{0}$ and matching condition). There exist $c_{0}^{\prime \prime}>c_{0}>0$ and a continuous function $\omega: \mathbb{R}^{n_{0}} \times$ $\mathbb{R}^{n_{1}} \rightarrow \mathbb{R}_{\geq 0}$, satisfying $\omega\left(\Theta\left(\zeta_{1}\right), \zeta_{1}\right)=0$ for all $\zeta_{1} \in \mathcal{Z}_{1}$, with $\Theta$ defined in the Standing Assumption, such that the following holds.

(i) The function $\omega$ satisfies

$$
\begin{aligned}
\sup \left\{\omega\left(\zeta_{0}, \zeta_{1}\right):\left(x, \zeta_{0}, \zeta_{1}\right) \in \mathcal{X} \times \mathcal{Z}_{0} \times \mathcal{Z}_{1}\right. \\
\left.V_{0}\left(x, \zeta_{0}\right) \leq \varepsilon_{0}, V_{1}\left(x, \zeta_{1}\right) \leq \varepsilon_{1}^{\prime}\right\} \leq c_{0},
\end{aligned}
$$

with $\varepsilon_{0}$ given in Assumption 1 and $\varepsilon_{1}^{\prime}$ given in Assumption 6.

(ii) The function $\omega$ satisfies

$\sup \left\{V_{0}\left(x, \zeta_{0}\right):\left(x, \zeta_{0}, \zeta_{1}\right) \in \mathcal{X} \times \mathcal{Z}_{0} \times \mathcal{Z}_{1}\right.$ $\left.V_{1}\left(x, \zeta_{1}\right) \leq \varepsilon_{1}^{\prime}, \omega\left(\zeta_{0}, \zeta_{1}\right) \leq c_{0}^{\prime \prime}\right\} \leq \varepsilon_{0}^{\prime}$, with $\varepsilon_{0}^{\prime}$ given in Assumption 1 and $\varepsilon_{1}^{\prime}$ given in Assumption 6.
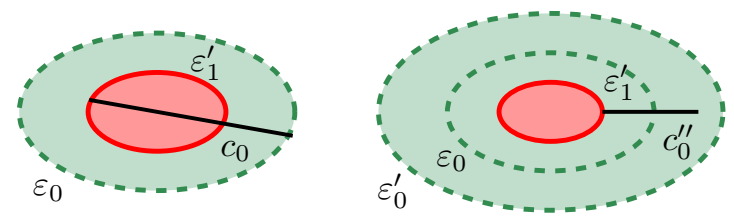

Fig. 4: Graphical interpretation of Assumption 7. Item (i) on the left, and item (ii) on the right. Dashed green line: level sets of $V_{0}\left(x, \zeta_{0}\right)$. Red line: level sets of $V_{1}\left(x, \zeta_{1}\right)$. Black line: value of the function $\omega\left(\zeta_{0}, \zeta_{1}\right)$.

The function $\omega$ in Assumption 7 is needed to measure the distance between the estimates provided by the two observers and is typically selected as $\omega\left(\zeta_{0}, \zeta_{1}\right)=\left|\hat{x}_{0}-\hat{x}_{1}\right|^{2}$. Recall that the set $\left\{\left(x, \zeta_{0}\right): V_{0}\left(x, \zeta_{0}\right) \leq \varepsilon_{0}^{\prime}\right\}$, given in Assumption 1, defines the domain of attraction of local observer (2), while the set $\left\{\left(x, \zeta_{1}\right): V_{1}\left(x, \zeta_{1}\right) \leq \varepsilon_{1}^{\prime}\right\}$, that can be overestimated via the norm estimator (9) according to Assumption 4, contains the asymptotic trajectories of global observer (3), see Assumption 3. As a consequence, item (i) of Assumption 7 states that, if the states of local and global observers reached their asymptotic behaviours, namely $\left\{\left(x, \zeta_{0}, \zeta_{1}\right): V_{0}\left(x, \zeta_{0}\right) \leq\right.$ $\left.\varepsilon_{0}, V_{1}\left(x, \zeta_{1}\right) \leq \varepsilon_{1}^{\prime}\right\}$, then the value of $\omega$ is small. On the other hand, item (ii) of Assumption 7 states that, if the value of $\omega$ is small enough and the global observer reached its asymptotic behaviour, then the state of the local observer needs to be in its domain of attraction $\left\{\left(x, \zeta_{0}\right): V_{0}\left(x, \zeta_{0}\right) \leq \varepsilon_{0}^{\prime}\right\}$. An illustration of items (i) and (ii) of Assumption 7, in the case in which $V_{0}$, $V_{1}$ are quadratic Lyapunov function, is depicted in Figure 4.

Remark 3 (Differences between Assumption 2 and Assumption 7). Assumption 7 may not hold if the set $\left\{\left(x, \zeta_{1}\right)\right.$ : $\left.V_{1}\left(x, \zeta_{1}\right) \leq \varepsilon_{1}^{\prime}\right\}$ is not small enough with respect to the sets $\left\{\left(x, \zeta_{0}\right): V_{0}\left(x, \zeta_{0}\right) \leq \varepsilon_{0}\right\},\left\{\left(x, \zeta_{0}\right): V_{0}\left(x, \zeta_{0}\right) \leq \varepsilon_{0}^{\prime}\right\}$, thus being sometimes more conservative than Assumption 2. On the other hand, the advantage of this second solution is that it does not need Assumption 2 to hold, namely we do not need the existence of a global norm estimator for $V_{0}$, which may be difficult to establish in some cases.

Under previous assumptions, the uniting observer (4) is given as in (11) with ${ }^{8}$

$$
\begin{aligned}
F(\xi, u, y):= & (1-q) \varphi_{0}\left(\zeta_{0}, u, y\right), \varphi_{1}\left(\zeta_{1}, u, y\right), \\
& (1-q)\left(-z_{0}+\omega\left(\vartheta_{0}\left(\zeta_{0}\right), \vartheta_{1}\left(\zeta_{1}\right)\right),\right. \\
& \left.-b_{1} z_{1}+\rho_{1}\left(y, h\left(\vartheta_{1}\left(\zeta_{1}\right), 0\right)\right), q, 0\right) .
\end{aligned}
$$

The following theorem can finally be stated.

Theorem 2. Suppose Assumption 1 and 3 to 7 hold. Let $T \in$ $[0, \infty), c_{0}^{\prime} \in\left(c_{0}, c_{0}^{\prime \prime}\right), c_{1}^{\prime} \in\left(c_{1}, c_{1}^{\prime \prime}\right)$, with $c_{1}^{\prime \prime}=\frac{\varepsilon_{1}^{\prime}-v_{1}}{a_{1}}$, where $c_{0}, c_{0}^{\prime \prime}, \varepsilon_{1}^{\prime}, v_{1}, a_{1}$ are given in Assumptions 4, 6 and 7. Then the hybrid observer (4), with $\xi, G, H, \mathcal{C}, \mathcal{D}$ selected as in (11), and $F$ as in (12), solves the uniting problem in which $\gamma=\gamma_{0}$

\footnotetext{
${ }^{8}$ With respect to the design proposed in [30], we use here two different estimators, $z_{0}$ and $z_{1}$, instead of using only one norm estimator driven by a function that depends on both $\rho_{1}$ and $\omega$ when $q=0$. The two approaches are essentially the same, but we prefer, here, to follow the two-norm estimator route to be consistent with the solution proposed in Section III-C.
} 
and $\mathcal{B}:=\left\{(x, \xi) \in \mathcal{X} \times \mathcal{O}: V_{0}\left(x, \zeta_{0}\right) \leq \varepsilon_{0}, \quad V_{1}\left(x, \zeta_{0}\right) \leq\right.$ $\left.\varepsilon_{1}, z_{0} \in\left[0, c_{0}\right], z_{1} \in[0, \infty), \tau \in[0, \infty), q=0\right\}$, where $\gamma_{0}, V_{0}$ are given by Assumption 1 , and $V_{1}$ by Assumption 3 .

\section{CASE STUdies}

We illustrate in this section how the results of Section III can be applied to combine an EKF with different types of global observers.

\section{A. Uniting an EKF with a global asymptotic observer}

Consider system (1), and suppose that $f, h$ are $C^{2}$ in their arguments, and moreover there exist $L_{x}, L_{w}>0$ such that the following condition holds

$$
\left|h\left(x_{1}, w\right)-h\left(x_{2}, 0\right)\right| \leq L_{x}\left|x_{1}-x_{2}\right|+L_{w}|w|
$$

for all $x_{1}, x_{2} \in \mathcal{X} \cup \vartheta_{0}\left(\mathcal{Z}_{0}\right) \cup \vartheta_{1}\left(\mathcal{Z}_{1}\right)$ and all $w \in \mathcal{W}$, where $\mathcal{W} \subseteq\left\{w \in \mathbb{R}^{n_{w}}:|w| \leq \bar{w}\right\}$, for some $\bar{w}$ which is selected according to the properties of the local observer.

We consider the case in which local observer (2) is an EKF (see, for instance, Definition 13 in [20, Section 1.3.1], or [5]) of the form

$$
\begin{aligned}
& \dot{\hat{x}}_{0}=f\left(\hat{x}_{0}, u\right)+K_{0}\left(\zeta_{0}\right)\left(y-\hat{y}_{0}\right) \\
& \dot{P}_{0}=J_{0}\left(\hat{x}_{0}, u\right) P_{0}+P_{0} J_{0}^{\top}\left(\hat{x}_{0}, u\right)+Q_{0} \\
& \quad-P_{0} C_{0}\left(\hat{x}_{0}\right)^{T} R_{0}^{-1} C_{0}\left(\hat{x}_{0}\right)^{T} P_{0} \\
& \hat{y}_{0}=h\left(\hat{x}_{0}, 0\right), \quad
\end{aligned}
$$

where $\zeta_{0}=\left(\hat{x}_{0}, \operatorname{vec}\left(P_{0}\right)\right) \in \mathcal{Z}_{0} \subseteq \mathbb{R}^{n_{x}} \times \mathbb{R}^{n_{x}^{2}}$ is the state of the observer,

$$
\begin{gathered}
K_{0}\left(\zeta_{0}\right):=P_{0} C_{0}\left(\hat{x}_{0}\right)^{\top} R_{0}^{-1}, \\
C_{0}\left(\hat{x}_{0}\right):=\frac{\partial h}{\partial x}\left(\hat{x}_{0}, 0\right), \quad J_{0}\left(\hat{x}_{0}, u\right):=\frac{\partial f}{\partial x}\left(\hat{x}_{0}, u\right),
\end{gathered}
$$

with $C_{0}$ satisfying $\left|C\left(\hat{x}_{0}\right)\right| \leq \sigma_{0}$ for all $\hat{x}_{0} \in \mathcal{Z}_{0}$, for some $\sigma_{0} \in \mathbb{R}_{>0}$, and $R_{0}, Q_{0}$ are any real, symmetric, positive definite matrices of appropriate dimensions. As explained for instance in [4], in a deterministic and nonlinear setting, $Q_{0}^{-1}$ represents the confidence in the trusted model (1) while $R_{0}^{-1}$ the confidence in the measurements (1), while in stochastic theory $Q_{0}$ and $R_{0}$ represent the covariances of the respectively drift Gaussian noise and measurement Gaussian noise. The next assumption states that (14) is a local asymptotic observer for system (1).

Assumption 8. There exist $V_{0}: \mathbb{R}^{n_{x}} \times \mathbb{R}^{n_{0}} \rightarrow \mathbb{R}_{\geq 0}$ and $\varepsilon_{0}, \varepsilon_{0}^{\prime}, \bar{w}>0$, such that Assumption 1 holds. Moreover, the function $V_{0}$ is selected as

$$
V_{0}\left(x, \zeta_{0}\right):=\left(x-\hat{x}_{0}\right)^{\top} P_{0}^{-1}\left(x-\hat{x}_{0}\right)
$$

and $P_{0}$ satisfies

$$
\underline{p}_{0} I \leq P_{0}(t) \leq \bar{p}_{0} I
$$

for all $t \geq 0$ along any solution pair $\left(\left(x, \zeta_{0}\right),(u, w)\right) \in \mathcal{X} \times$ $\mathcal{Z}_{0} \times \mathcal{U} \times \mathcal{W}$ to system (1), (14).

Conditions under which Assumption 8 is verified can be found in e.g., [4], [5]. In particular, the choice of $V_{0}$ arises naturally in the literature of EKF. Moreover, boundedness of $P_{0}(t)$ along any trajectory of (1), (14), is verified when system

\begin{tabular}{ll}
\hline$\mu_{0}=2 \varrho_{0} L_{x}^{2} \bar{p}_{0}$ & $a_{0}=\left(\underline{p}_{0} \underline{\alpha}_{1}\right)^{-1}$ \\
$\mu_{0}^{\prime}=2 \varrho_{0} L_{w}^{2}$ & $b_{0}=\frac{1}{2} \lambda_{1}$ \\
$v_{0}=a_{0} b_{0}^{-1} \psi_{1}(\bar{w})$ & $c_{0}=\max \left\{a_{0}^{-1} \varepsilon_{0}, b_{0}^{-1}\left(\mu_{0} \varepsilon_{0}+\mu_{0}^{\prime} \bar{w}^{2}\right)\right\}$ \\
$\rho_{0}\left(y, \hat{y}_{0}\right)=\varrho_{0}\left|y-\hat{y}_{0}\right|^{2}$ & $\varrho_{0}=\left(k_{0}+k_{1}\right)^{2} k_{2}^{2}\left(2 \lambda_{1} \underline{\alpha}_{1}\right)^{-1}$ \\
$k_{0}=\bar{p}_{0} \sigma_{0}\left|R_{0}^{-1}\right|$ & \\
\hline$\varepsilon_{1}^{\prime}=2 \underline{\alpha}_{1} \bar{p}_{0} \varepsilon_{0}$ & $a_{1}=1$ \\
$\varepsilon_{1} \in\left(v_{1}, \varepsilon_{1}^{\prime}-v_{1}\right)$ & $b_{1}=\lambda_{1}$ \\
$v_{1}=b_{1}^{-1} \psi_{1}(\bar{w})$ & $c_{1}=\varepsilon_{1}$ \\
$\rho_{1}\left(y, \hat{y}_{1}\right)=\varrho_{1}\left|y-\hat{y}_{1}\right|^{2}$ & $\varrho_{1} \in\left(0, b_{1} c_{1}\left(L_{x} \sqrt{\frac{\varepsilon_{1}}{\underline{\underline{\alpha}}_{1}}}+L_{w} \bar{w}\right)^{-2}\right]$, \\
\hline
\end{tabular}

TABLE I: Uniting EKF (14) and global asymptotic observer (15). Design of the parameters satisfying Assumptions 1-5 in Proposition 1.

(1) is uniformly observable, see e.g., [4], [5] or [20, Section 1.3.1]. The robustness properties of the EKF with respect to measurement noise $w$ have been discussed in [4].

The global observer (3) is of the form

$$
\dot{\hat{x}}_{1}=f\left(\hat{x}_{1}, u\right)+K_{1}\left(\hat{x}_{1}\right)\left(y-\hat{y}_{1}\right), \quad \hat{y}_{1}=h\left(\hat{x}_{1}, 0\right),
$$

where $\hat{x}_{1}=\zeta_{1} \in \mathcal{Z}_{1} \subseteq \mathbb{R}^{n_{x}}$ is the state of the observer and $K_{1}$ satisfies $\left|K_{1}\left(\hat{x}_{1}\right)\right| \leq k_{1}$ for all $\hat{x}_{1} \in \mathcal{Z}_{1}$ for some $k_{1} \in \mathbb{Z}_{>0}$. We also suppose that the projection of $\mathcal{Z}_{0}$ on $\mathbb{R}^{n_{x}}$ coincides with $\mathcal{Z}_{1}$. The observer (15) satisfies the next assumption.

Assumption 9 (Global asymptotic observer). There exist a continuous function $V_{1}: \mathcal{X} \times \mathcal{Z}_{1} \rightarrow \mathbb{R}_{\geq 0}, \psi_{1} \in \mathcal{K}$, and $\bar{\alpha}_{1}, \underline{\alpha}_{1}, \lambda_{1}, k_{2}>0$, such that the following holds

$$
\begin{gathered}
\underline{\alpha}_{1}\left|x-\hat{x}_{1}\right|^{2} \leq V_{1}\left(x, \hat{x}_{1}\right) \leq \bar{\alpha}_{1}\left|x-\hat{x}_{1}\right|^{2} \\
\left|\nabla V_{1}\left(x, \hat{x}_{1}\right)\right| \leq k_{2}\left|x-\hat{x}_{1}\right| \\
\left\langle\nabla V_{1}\left(x, x_{1}\right), \Phi_{1}\left(x, u, w, \hat{x}_{1}\right)\right\rangle \leq-\lambda_{1} V_{1}\left(x, \hat{x}_{1}\right)+\psi_{1}(|w|)
\end{gathered}
$$

for all $\left(x, \hat{x}_{1}\right) \in \mathcal{X} \times \mathcal{Z}_{1}$ and $u \in \mathcal{U}$, where

$\Phi_{1}\left(x, u, w, \hat{x}_{1}\right):=\left(f(x, u), f\left(\hat{x}_{1}, u\right)+K_{1}\left(\hat{x}_{1}\right)\left(h(x, w)-h\left(\hat{x}_{1}, 0\right)\right)\right.$.

Assumption 9 states that (3) is a global ${ }^{9}$ asymptotic observer for system (1). In particular, the function $V_{1}$ is a Lyapunov function, typically quadratic, ensuring the global exponential input-to-state stability of the associated estimation error system with input $w$. Depending on the properties of $f, h$, the output injection gain $K_{1}$ in (15) and the function $V_{1}$ ensuring the conditions in Assumption 9 can be designed with various techniques. For instance, when $f, h$ are expressed in the canonical observability form, we can design (15) by following the HGO design proposed in [2], [20]. When $f, h$ are expressed in different coordinates but are diffeomorphic to the observability canonical form, we can follow the design proposed in [22], [27] and references therein. When $f, h$ are Lipschitz on $\mathcal{X} \times \mathcal{U}$, it is also possible to follow the techniques proposed in e.g., [15], [26], [33]. Finally, other approaches, such as [7], [21], [23], [24], [27], in which $n_{1}>n_{x}$, can be used by slightly modifying the conditions of Assumption 9 (and therefore the computation of the parameters given in Table I).

With the definition of observers (14) and (15), we select the function $\Theta$ of the Standing Assumption as $\Theta\left(\zeta_{1}\right):=\left(\zeta_{1}, \bar{P}_{0}\right)$,

\footnotetext{
${ }^{9}$ Global with respect to the sets $\mathcal{X} \times \mathcal{Z}_{1}$.
} 
where $\bar{P}_{0} \in \mathbb{R}^{n_{x}^{2}}$ is any positive definite symmetric matrix satisfying $\underline{p}_{0} \leq \bar{P}_{0} \leq \bar{p}_{0}$, with $\underline{p}_{0}, \bar{p}_{0}$ given by Assumption 8 . Finally, the following result can be stated.

Proposition 1. Consider system (1), EKF (14) and global observer (15). Suppose Assumptions 8 and 9 hold and, moreover, suppose that the following conditions hold

$$
\begin{aligned}
\varepsilon_{0}>2 \underline{\alpha}_{1}^{-1} \bar{p}_{0}^{-1} v_{1}, \\
\varepsilon_{0}^{\prime}>v_{0}+\max \left\{\varepsilon_{0}, \frac{a_{0}}{b_{0}}\left(\mu_{0} \varepsilon_{0}+\mu_{0}^{\prime} \bar{w}^{2}\right)\right\}
\end{aligned}
$$

where $\varepsilon_{0}, \varepsilon_{0}^{\prime}, \bar{p}_{0}$ are given by Assumption $8, \underline{\alpha}_{1}$ is given by Assumption 9, and $a_{0}, b_{0}, \mu_{0}, \mu_{0}^{\prime}, v_{0}, v_{1}$ are selected as in Table I. Then, Assumptions 1 to 5 hold with the parameters given in Table I.

According to the previous proposition, we can therefore unite EKF (14) and observer (15) by using the design proposed in Theorem 1.

Remark 4 (On the conditions of Proposition 1). The conditions (17) given in the statement of the proposition are of qualitative nature, and in general only sufficient. Moreover, they may be conservative, since they are computed by using conservative inequalities. These conditions depend in general on the value of the measurement noise $\bar{w}$, see how $v_{0}, v_{1}$ are selected in Table I. When no measurement noise is present, namely $\bar{w}=0$, conditions (17) reduce to $\varepsilon_{0}>0$ and $\varepsilon_{0}^{\prime}>\max \left\{1, \frac{a_{0}}{b_{0}} \mu_{0}\right\} \varepsilon_{0}$. Finally, note that if EKF (14) has a global domain of attraction, namely $\varepsilon_{0}, \varepsilon_{0}^{\prime}$ can be chosen arbitrarily large, then conditions (17) are always satisfied.

Remark 5 (On the parameters of Table I). In order to implement the hybrid observer (11), it is not needed to compute all the parameters defined in Table I. In particular, we can always select ${ }^{10} a_{0}=b_{0}=a_{1}=b_{1}=1$ and focus on the tuning of the parameters $c_{0}^{\prime}, c_{1}^{\prime}, \varrho_{0}, \varrho_{1}$ which can be estimated via numerical simulations. A numerical example is given in Section V.

Remark 6 (Comparison with other approaches). The problem of uniting an EKF with a HGO, for systems which are expressed in the observability canonical form, has been addressed with a different technique in [7]. Therein, the two observers are merged into a single observer by making vary a single parameter $\theta$. The resulting observer behaves as a HGO when $\theta$ is large, and as an EKF, when $\theta$ is small. Similarly to the construction of this work, the proposed continuous-time adaptive mechanism to vary $\theta$ is based on a norm estimator of the estimation error $|x-\hat{x}|$, see [7, Lemma 4].

\section{B. Uniting an EKF with a global approximate observer}

We consider again system (1) where $f, h$ are $C^{2}$ and verify (13), and the measurement noise ranges in some set $\mathcal{W} \subseteq$ $\left\{w \in \mathbb{R}^{n_{w}}:|w| \leq \bar{w}\right\}$, for some $\bar{w} \in \mathbb{R}_{>0}$. The local observer is still given by EKF (14) and we suppose that Assumption 8 holds.

We consider in this section the case in which we only know how to design a global asymptotic observer (in the absence of

\footnotetext{
${ }^{10}$ Recall that the existence of the Lyapunov function $V_{1}$ in Assumption 9 implies the existence of another Lyapunov function with arbitrary $\lambda_{1}$. See [12], [35]
}

noise $w$ ) for an approximation of system (1). In particular, we write

$$
f(x, u):=f_{a}(x, u)+\left(f(x, u)-f_{a}(x, u)\right)
$$

and

$$
h(x, w):=h_{a}(x)+\left(h(x, w)-h_{a}(x)\right),
$$

where $f_{a}$ is an approximation of $f$ and $h_{a}$ is an approximation of $h$, and we assume that we know how to design a global asymptotic observer for system

$$
\dot{x}=f_{a}(x, u), \quad y=h_{a}(x) .
$$

The observer is selected as

$$
\dot{\hat{x}}_{1}=f_{a}\left(\hat{x}_{1}, u\right)+K_{1}\left(\hat{x}_{1}\right)\left(y-\hat{y}_{1}\right), \quad \hat{y}_{1}=h_{a}\left(\hat{x}_{1}\right),
$$

where $\hat{x}_{1} \in \mathcal{Z}_{1} \subseteq \mathbb{R}^{n_{x}}$ is the state and $K_{1}$ satisfies $\left|K_{1}\left(\hat{x}_{1}\right)\right| \leq$ $k_{1}$ for all $\hat{x}_{1} \in \mathcal{Z}_{1}$ for some $k_{1} \in \mathbb{Z}_{>0}$. It satisfies the next assumption.

Assumption 10 (Approximate observer). There exist a continuous function $V_{1}: \mathcal{X} \times \mathcal{Z}_{1} \rightarrow \mathbb{R}_{\geq 0}$, and $\bar{\alpha}_{1}, \underline{\alpha}_{1}, \lambda_{1}, k_{2}>0$, such that the following holds

$$
\begin{gathered}
\underline{\alpha}_{1}\left|x-\hat{x}_{1}\right|^{2} \leq V_{1}\left(x, \hat{x}_{1}\right) \leq \bar{\alpha}_{1}\left|x-\hat{x}_{1}\right|^{2} \\
\left|\nabla V_{1}\left(x, \hat{x}_{1}\right)\right| \leq k_{2}\left|x-\hat{x}_{1}\right| \\
\left\langle\nabla V_{1}\left(x, x_{1}\right), \Phi_{a}\left(x, u, \hat{x}_{1}\right)\right\rangle \leq-\lambda_{1} V_{1}\left(x, \hat{x}_{1}\right)
\end{gathered}
$$

for all $\left(x, \hat{x}_{1}\right) \in \mathcal{X} \times \mathcal{Z}_{1}$ and $(u, w) \in \mathcal{U} \times \mathcal{W}$, where

$\Phi_{a}\left(x, u, \hat{x}_{1}\right):=\left(f_{a}(x, u), f_{a}\left(\hat{x}_{1}, u\right)+K_{1}\left(\hat{x}_{1}\right)\left(h_{a}(x)-h_{a}\left(\hat{x}_{1}\right)\right)\right.$.

Assumption 10 states that (19) is a global ${ }^{11}$ asymptotic observer for system (18) in absence of measurement noise, namely when $w=0$. Observer (19) may be designed by following any of the techniques proposed in e.g., [2], [15], [20], [22], [26], [27]. When considering observers with $n_{1}>n_{x}$, also interval observers [34] can be combined with the EKF (14). The design of observer (19) obviously depends on the approximate model (18). This can be obtained by linearising $f, h$ around an equilibrium (if any), or by ignoring some dynamics of $f, h$, which complicate if not obstruct the design of a global asymptotic observer for the original system (1). Furthermore, we assume that the functions $f_{a}, h_{a}$ are locally Lipschitz and that the mismatch between the functions $f, h$ and $f_{a}, h_{a}$ is globally bounded on $\mathcal{X} \times \mathcal{U}$, namely there exist $\Delta_{f}, \Delta_{h} \geq 0$ such that

$$
\left|f(x, u)-f_{a}(x, u)\right| \leq \Delta_{f}, \quad\left|h(x, 0)-h_{a}(x)\right| \leq \Delta_{h},
$$

for all $(x, u) \in \mathcal{X} \times \mathcal{U}$. Hence, when the sets $\mathcal{X}, \mathcal{U}$ are compact $^{12}$, condition (21) is satisfied for any function pair $\left(f_{a}, h_{a}\right)$ globally bounded on $\mathcal{X}, \mathcal{U}$.

We select the functions $\Theta$ satisfying the Standing Assumptions as in Section IV-A, namely $\Theta\left(\zeta_{1}\right):=\left(\zeta_{1}, \bar{P}_{0}\right)$, where $\bar{P}_{0} \in \mathbb{R}^{n_{x}^{2}}$ is any positive definite symmetric matrix satisfying $\underline{p}_{0} \leq \bar{P}_{0} \leq \bar{p}_{0}$, with $\underline{p}_{0}, \bar{p}_{0}$ given by Assumption 8. The following result holds.

\footnotetext{
${ }^{11}$ Global with respect to the sets $\mathcal{X} \times \mathcal{Z}_{1}$.

${ }^{12}$ This case occurs when input and plant dynamics are ultimately bounded, like in the case of a limit cycle.
} 


\begin{tabular}{ll}
\hline$\omega\left(\zeta_{0}, \zeta_{1}\right)=\left|\hat{x}_{0}-\hat{x}_{1}\right|^{2}$ & $c_{0}=4 \bar{p}_{0} \varepsilon_{0}$ \\
$c_{0}^{\prime \prime}=\frac{1}{2}\left(\underline{p}_{0} \varepsilon_{0}^{\prime}-2 \bar{p}_{0} \varepsilon_{0}\right)$ & \\
\hline$\varepsilon_{1}^{\prime}=\underline{\alpha}_{1} \bar{p}_{0} \varepsilon_{0}$ & $a_{1}=1$ \\
$\varepsilon_{1} \in\left(v_{1}, \varepsilon_{1}^{\prime}-v_{1}\right)$ & $b_{1}=\frac{1}{2} \lambda_{1}$, \\
$v_{1}=k_{2}^{2}\left(\Delta_{f}+k_{1} \Delta_{h}+k_{1} L_{w} \bar{w}\right)^{2}\left(\underline{\alpha}_{1} \lambda_{1}^{2}\right)^{-1}$, & $c_{1}=\varepsilon_{1}$ \\
$\rho_{1}\left(y, \hat{y}_{1}\right):=\varrho_{1}\left|y-\hat{y}_{1}\right|^{2}$ & \\
$\varrho_{1} \in\left(0, b_{1} c_{1}\left(L_{x} \sqrt{\varepsilon_{1} \underline{\alpha}_{1}^{-1}}+L_{w} \bar{w}+\Delta_{h}\right)^{-2}\right]$ & \\
\hline
\end{tabular}

TABLE II: Uniting EKF (14) and global approximate observer (19). Design of the parameters satisfying Assumptions 1, 3-7 in Proposition 2.

Proposition 2. Consider system (1), EKF (14), and global observer (19). Suppose Assumptions 8 and 10 hold and, moreover, suppose that the following conditions hold

$$
\varepsilon_{0}>2 \underline{\alpha}_{1}^{-1} \bar{p}_{0}^{-1} v_{1}, \quad \varepsilon_{0}^{\prime}>10 \bar{p}_{0} \underline{p}_{0}^{-1} \varepsilon_{0},
$$

where $\varepsilon_{0}, \varepsilon_{0}^{\prime}, \bar{p}_{0}, \underline{p}_{0}$ are given by Assumption $8, \underline{\alpha}_{1}$ is given in Assumption 1 $\overline{0}$, and $v_{1}$ is defined in Table II. Then, Assumption 1 and Assumptions 3 to 7 hold, with the parameters chosen as in Table II.

According to Proposition 2, we can therefore unite EKF (14) and observer (19) by using the design proposed in Theorem 2 . As for Proposition 1, it is worth noticing that the conditions (22) may be very conservative and it is therefore to be appreciated the qualitative nature of the result.

\section{NumericAl EXAMPLE}

We apply the result of Proposition 1 in Section IV to unite an EKF, see e.g., [4], with a HGO, see e.g., [2], for a perturbed Duffing oscillator, see [30], described as

$$
\dot{x}=A x+B \phi(x)+Q_{c} v, \quad y=C x+R_{c} w,
$$

where $x=\left(x_{a}, x_{b}\right)^{\top} \in \mathbb{R}^{2}$ is the state, $y \in \mathbb{R}$ is the output, $\phi(x)=x_{a}-x_{a}^{3}$, and $(A, B, C)$ is a triplet in prime form, namely

$$
A=\left(\begin{array}{ll}
0 & 1 \\
0 & 0
\end{array}\right), \quad B=\left(\begin{array}{l}
0 \\
1
\end{array}\right), \quad C=\left(\begin{array}{ll}
1 & 0
\end{array}\right),
$$

In (23), the signal $w \in \mathbb{R}$ is the measurement noise, $v \in \mathbb{R}^{2}$ is some unmeasured disturbance affecting the plant, and the matrices $Q_{c}, R_{c}$ are unknown. Although the theory in Section III is not explicitly developed to treat dynamic disturbances, we consider here the effect of $v$ in order to test the robustness of the proposed uniting observer. For any initial condition $x(0)$ ranging in some given compact set $\mathcal{X}^{\circ} \subset \mathbb{R}^{2}$, and for any (small enough) bounded input $v$, there exists a compact set $\mathcal{X} \supseteq \mathcal{X}^{\circ}$, which is forward invariant for (23), which can be numerically determined.

By following [4], the local asymptotic observer in (2) is (14), which gives here

$$
\begin{aligned}
& \dot{\hat{x}}_{0}=A \hat{x}_{0}+B \phi\left(\hat{x}_{0}\right)+K_{0}\left(\zeta_{0}\right)\left(y-C \hat{x}_{0}\right) \\
& \dot{P}_{0}=J_{0}\left(\hat{x}_{0}\right) P_{0}+P_{0} J_{0}\left(\hat{x}_{0}\right)^{T}-P_{0} C^{T} R_{0}^{-1} C P_{0}+Q_{0} \\
& \hat{y}_{0}=C \hat{x}_{0}
\end{aligned}
$$

where $\hat{x}_{0}=\left(\hat{x}_{0 a}, \hat{x}_{0 b}\right) \in \mathbb{R}^{2}$ is the estimate, $P_{0} \in \mathbb{R}^{2 \times 2}$, $\zeta_{0}=\left(\hat{x}_{0}, \operatorname{vec}\left(P_{0}\right)\right)$ is the overall state,

$$
J_{0}\left(\hat{x}_{0}\right):=A+B \frac{\partial \phi\left(\hat{x}_{0}\right)}{\partial x}, \quad K_{0}\left(\zeta_{0}\right)=P_{0} C^{T} R_{0}^{-1},
$$

and $Q_{0}, R_{0}$ are positive definite matrices. Since system (23) is uniformly observable, the set $\mathcal{X}$ is forward invariant for (23) and the second derivative of $\phi$ is bounded on compact sets, it can be proved, by following [5, Theorem 1.1.1], that EKF (24) is a local observer satisfying Assumption 1 and the matrix $P_{0}$ in (24) verifies Assumption 8 for any $P_{0}(0)=P_{0}(0)^{\top}>0$. Note that obtaining non-conservative estimates of the basin of attraction for EKF designs is in general hard. As a consequence, in the following, we selected various initial conditions for observer (24) and convergence has been verified by simulations for each of these initial conditions. The motivation for the uniting observer here is that, although the EKF seems to globally converge, very slow transients occur when the initial estimation $\left|x(0)-\hat{x}_{0}(0)\right|$ is large, as shown in Figure 5. The global observer in (3) is given by a HGO designed with state $\zeta_{1}=\hat{x}_{1}$ and dynamics given by

$$
\begin{aligned}
& \dot{\hat{x}}_{1}=A \hat{x}_{1}+B \phi_{s}\left(\hat{x}_{1}\right)+D_{\kappa} L_{1}\left(y-C \hat{x}_{1}\right) \\
& \hat{y}_{1}=C \hat{x}_{1},
\end{aligned}
$$

where $\hat{x}_{1}=\left(\hat{x}_{1 a}, \hat{x}_{1 b}\right) \in \mathbb{R}^{2}$ is the observer state, $L_{1} \in$ $\mathbb{R}^{2 \times 1}$ is any matrix chosen such that $A-L_{1} C$ is Hurwitz, $D_{\kappa}=\operatorname{diag}\left(\kappa, \kappa^{2}\right)$, where $\kappa \geq 1$ is the so called high-gain parameter, and the function $\phi_{s}(\cdot):=\operatorname{sat}_{r}(\phi(\cdot))$ where $\operatorname{sat}_{r}$ is any (continuous) saturation function with saturation level $r \geq \max _{x \in \mathcal{X}}|\phi(x)|$. Given the compact set $\mathcal{X}$ in (23), the observer (25) is a global asymptotic observer for (23) for $\kappa$ large enough. Assumption 9 can be established with the Lyapunov function

$$
V_{1}=\hat{e}_{1}^{T} H \hat{e}_{1}
$$

where $H$ is solution of

$$
H\left(A-L_{1} C\right)+\left(A-L_{1} C\right)^{T} H=-I
$$

and $\hat{e}_{1}=\kappa D_{k}^{-1}\left(\hat{x}_{1}-x\right)$, see [2]. In view of Proposition 1, we can therefore unite EKF (24) and HGO (25) and design observer (11) satisfying Theorem 1. For this, we select $\rho_{i}\left(y, \hat{y}_{i}\right):=\left|y-\hat{y}_{i}\right|, b_{i}=1, i=\{0,1\}$, $\Theta\left(\zeta_{1}\right)=\left(\hat{x}_{1}, \operatorname{vec}\left(I_{2 \times 2}\right)\right)^{T}$, and $\varphi_{0}, \varphi_{1}$ chosen accordingly to the definitions of (24), (25).

In the simulations, $v$ and $w$ are generated by $\dot{\eta}_{i}=\omega_{i} S \eta_{i}$, $i=\{1,2,3\}, v=Q_{1}+\eta_{1}+Q_{2} \eta_{2}, w=Q_{3} \eta_{3}$, where $S:=\left(\begin{array}{cc}0 & 1 \\ -1 & 0\end{array}\right)$, and the matrices $Q_{1}, Q_{2} \in \mathbb{R}^{2 \times 2}$ and $Q_{3} \in \mathbb{R}^{1 \times 2}$ have unitary norms. We selected $\omega_{1}=3$, $\omega_{2}=27, \omega_{3}=32, Q_{1}=\left(\begin{array}{lll}0.99 & 0.12,-0.12 & 0.99\end{array}\right)$, $Q_{2}=\left(\begin{array}{lll}0.85 & 0.53,-0.53 & 0.85\end{array}\right), Q_{3}=\left(\begin{array}{ll}0.65 & 0.76\end{array}\right), Q_{c}=$ $\operatorname{diag}(0.1,0.1), R_{c}=0.5, Q_{0}=\operatorname{diag}(0.2,0.2), R_{0}=1$. The initial conditions of (23) range in the compact set $\mathcal{X}^{\circ}:=\left\{\left(x_{a}, x_{b}\right) \in \mathbb{R}^{2}: x_{a}^{2}+x_{b}^{2} \leq 25\right\}$ and we have $\mathcal{X} \subset\left\{\left(x_{a}, x_{b}\right) \in \mathbb{R}^{2}:\left|x_{a}\right| \leq 6,\left|x_{b}\right| \leq 20\right\}$. This can be computed numerically. According to the bound, we selected $L_{1}=(1,1), r=210$ and $\kappa=15$. The constants $c_{0}^{\prime}, c_{1}^{\prime}, T$ 

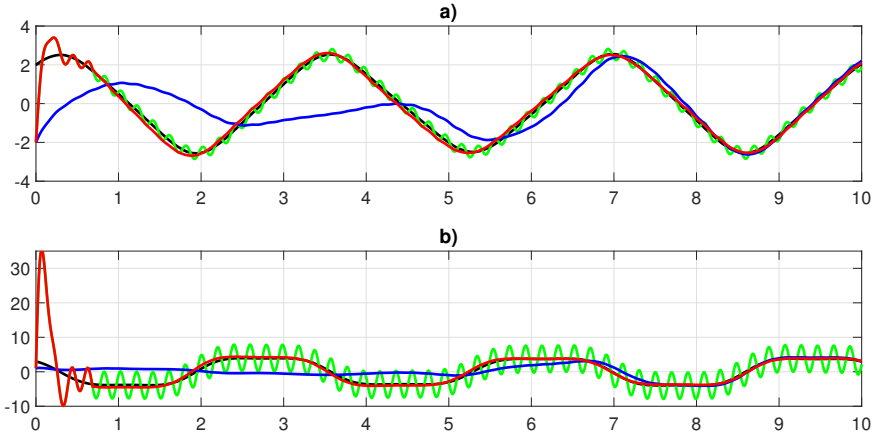

Fig. 5: State $x$ of the Duffing oscillator (black), state $\hat{x}_{0}$ of EKF (blue), state $\hat{x}_{1}$ of $\mathrm{HGO}$ (green), and estimate $\hat{x}$ of the uniting observer (red). Figure a) First components of $x, \hat{x}_{0}, \hat{x}_{1}, \hat{x}$. Figure b) Second components of $x, \hat{x}_{0}, \hat{x}_{1}, \hat{x}$.

are tuned via numerical simulations in order to improve the performances of the resulting uniting observer.

We have selected the following set of initial conditions $x(0,0)=(2,3)$, for $(23)$, and $\hat{x}_{0}(0,0)=(-2,1), P(0,0)=$ $I_{2 \times 2}, \quad \hat{x}_{1}(0,0)=(-2,1), \quad z_{0}(0,0)=0, z_{1}(0,0)=1$ $q(0,0)=1, \tau(0,0)=0$ for $\xi(0,0)$ in (11c). The parameter $T=0.01$ is selected small enough with respect to the dynamics of the HGO. In order to select the parameters $c_{0}^{\prime}, c_{1}^{\prime}$, we have run simulations and we have observed that the asymptotic behaviours of $z_{0}, z_{1}$ of (7), (9) oscillate around 0.35 . This indicates that the values of $c_{0}^{\prime}$ and $c_{1}^{\prime}$ cannot be chosen arbitrarily small.

Figure 5 shows the behaviours of system (23), observers (24), (25), and the hybrid observer (11) obtained by uniting the EKF and the HGO and by selecting $c_{0}^{\prime}=0.6, c_{1}^{\prime}=0.8$. As expected, the speed of convergence of the EKF (24) is very slow, and the asymptotic behaviour is quite accurate. On the contrary, the HGO (25) shows a fast convergence with poor asymptotic behaviour. The uniting observer (11) combines both behaviours by taking advantage of the fast convergence of HGO and the good steady-state behaviour of the EKF. We have then studied the influence of the parameters $c_{0}^{\prime}, c_{1}^{\prime}$ on the uniting observer (11) by picking different values, namely $c_{0}^{\prime}=0.4$ and $c_{1}^{\prime}=0.5$. Figure 6 shows the behaviours of the error coordinates $\hat{x}-x$ of (5) in the two cases. Note that, in the second simulation, the value of $c_{0}^{\prime}$ is too close to its expected asymptotic behaviour. As a consequence, more jumps occur during the transients, since the EKF dynamics has not the time to converge while the norm estimator $z_{0}$ exceeds the desired threshold $c_{0}^{\prime}$. Similarly, simulations indicate that a choice of $c_{0}^{\prime}$ too large produces largest transients, since norm estimator $z_{0}$ takes more time to detect whether the local observer (24) is close to the system state or not.

\section{PROOFS}

For the sake of compactness, throughout this section we will use the following compact notations $V_{0}(t, j):=$ $V_{0}\left(x(t, j), \zeta_{0}(t, j)\right), V_{1}(t, j):=V_{1}\left(x(t, j), \zeta_{1}(t, j)\right), \rho_{0}(t, j):=$ $\rho_{0}\left(y(t, j), \hat{y}_{0}(t, j)\right), \rho_{1}(t, j):=\rho_{1}\left(y(t, j), \hat{y}_{1}(t, j)\right), \omega(t, j):=$ $\omega\left(\hat{x}_{0}(t, j), \hat{x}_{1}(t, j)\right)$.
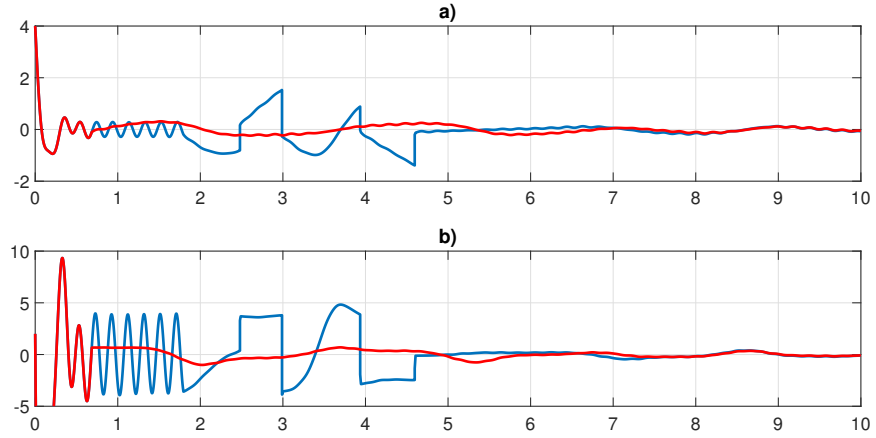

Fig. 6: Error coordinates $\hat{x}-x$ of the uniting observer for two different sets of values of $c_{0}^{\prime}, c_{1}^{\prime}$. Figure a) $\hat{x}_{a}-x_{a}$. Figure b) $\hat{x}_{b}-x_{b}$. Red line: $c_{0}^{\prime}=0.6, c_{1}^{\prime}=0.8$. Blue line: $c_{0}^{\prime}=0.4$, $c_{1}^{\prime}=0.5$.

\section{A. Proof of Theorem 1}

The proof follows by showing that items (a), (b), (c) of Definition 1 hold. The main steps are similar to those of the proof of Theorem 3.2 in [12], although non-trivial differences arise due to the features of the estimation problem.

(a) Completeness of solutions and finite number of jumps: Consider system (5), with $F, G, H, \mathcal{C}, \mathcal{D}$ selected as in (11). Completeness of solutions follows by direct application of Proposition 2.10 in [32]. First of all, the sets $\mathcal{X}, \mathcal{C}, \mathcal{D}, \mathcal{U}$ and $\mathcal{W}$ are closed, and $f, F, G$ are continuous. Moreover, in view of the Standing Assumption, the condition (VC) in Proposition 2.10 in [32] is verified. Then, recall that by definition of $F, G, \mathcal{C}, \mathcal{D}$ in (11b), (11c), (11d), we have $G\left(\mathcal{D}_{0}\right) \in \mathcal{C}_{1}$ and $G\left(\mathcal{D}_{1}\right) \in \mathcal{C}_{0}$. We deduce that $G(\mathcal{D}) \in \mathcal{C}$. As a consequence, item (c) in Proposition 2.10 in [32] cannot occur. Therefore, either the solutions ${ }^{13}$ are complete, or the time domain is bounded, because during flow, solutions cannot be extended, see conditions (a), (b) of Proposition 2.10 in [32]. Next, we want to show that also condition (b) can be ruled out, namely solutions need to be complete. For this, suppose there exists a solution $\xi$ that does not enter $\mathcal{D}$ and cannot be extended in $\xi \in \mathcal{C}$. This solution is either in $\mathcal{C}_{0}$ or in $\mathcal{C}_{1}$. When $\xi \in \mathcal{C}_{0}$, the flow of $\left(x, \zeta_{0}, \zeta_{1}\right)$ corresponds to solutions to (1), (2) and (3). In view of Standing Assumption, the components $\left(x, \zeta_{0}, \zeta_{1}\right)$ are complete. By continuity of $\rho_{0}, \rho_{1}$ the solutions to $z_{0}, z_{1}$ given by (7), (9) are complete. Finally, $\tau$ and $q$ remain constant on flows. As a consequence, any solution in $\mathcal{C}_{0}$ that does not jump must be complete. With similar arguments, any solution flowing in $\mathcal{C}_{1}$, which that does not jump, must be complete. As a consequence, condition (b) of Proposition 2.10 in [32] cannot occur and only (a) can hold, namely solution are complete. Now consider the following claim, whose proof is given in Section VI-B1.

Claim 1. Let $((x, \xi),(u, w))$ be a solution pair to (5). There does not exist an infinite non-decreasing sequence of hybrid

\footnotetext{
${ }^{13}$ Recall that we talk of maximal solutions throughout the paper, see Section II.
} 
times $\left(\left(t_{n}, j_{n}\right)_{n \in \mathbb{Z}_{>0}}\right) \in \operatorname{dom}(x, \xi)$, such that we have

$$
q\left(t_{2 n}, j_{2 n}\right)=0, \quad q\left(t_{2 n+1}, j_{2 n+1}\right)=1,
$$

for all $n \in \mathbb{Z}_{\geq 0}$.

Claim 1 states that the discrete variable $q$ cannot switch back and forth persistently between $q=0$ and $q=1$. As a consequence, the number of jumps must be finite. This completes the first part of the proof.

(b) Global convergence: We prove the global convergence property by combining Claim 1 with the next two claims. The first claim states that the discrete variable $q$ has to take the value 0 at some (hybrid) time. In other words, any solution $(x, \xi)$ to $(5)$ enters the set $\mathcal{C}_{0}$ at some (hybrid) time. The second claim states that, if the discrete variable $q$ remains equal to 0 after a sufficiently long time, i.e., $(x, \xi)$ remains in $\mathcal{C}_{0}$ for a sufficiently large time, then the asymptotic behaviour of hybrid observer (11) corresponds to that of local observer (2).

Claim 2. For any initial condition $(x(0,0), \xi(0,0))$ and any hybrid input $(u(t, j), w(t, j))$, the corresponding solution pair $((x, \xi),(u, w))$ to $(5)$ is such that there exists $(\bar{t}, \bar{j}) \in$ $\operatorname{dom}(x, \xi)$ with $q(\bar{t}, \bar{j})=0$.

Claim 3. Let $((x, \xi),(u, w))$ be a solution pair to (5). If there exists $(\bar{t}, \bar{j}) \in \operatorname{dom}(x, \xi)$, such that $q(t, j)=0$ for all $(t, j) \in$ $\operatorname{dom}(x, \xi)$, with $(t, j) \succeq(\bar{t}, \bar{j})$, then (6) holds.

We are in the position to combine the previous claims to prove the global convergence property. Pick any solution pair $((x, \xi),(u, w))$ to (5). In view of Claim 1, there exists a hybrid time $\left(\bar{t}_{1}, \bar{j}_{1}\right) \in \operatorname{dom}(x, \xi)$ such that either $q(t, j)=0$ or $q(t, j)=1$ for all $(t, j) \in \operatorname{dom}(x, \xi)$ with $(t, j) \succeq\left(\bar{t}_{1}, \bar{j}_{1}\right)$. By applying Claim 2 and invoking the semigroup property, we know that it cannot exist a hybrid time $\left(\bar{t}_{2}, \bar{j}_{2}\right) \in \operatorname{dom}(x, \xi),\left(\bar{t}_{2}, \bar{j}_{2}\right) \succeq\left(\bar{t}_{1}, \bar{j}_{1}\right)$ such that, $q(t, j)=1$, for all $(t, j) \in \operatorname{dom}(x, \xi)$ such that $(t, j) \succeq\left(\bar{t}_{2}, \bar{j}_{2}\right)$. As a result, the solution $(x, \xi)$ is such that $q(t, j)=0$ for all $(t, j) \in \operatorname{dom}(x, \xi),(t, j) \succeq\left(\bar{t}_{1}, \bar{j}_{1}\right)$. Therefore, by applying Claim 3 we have that (6) holds. This concludes the global convergence property.

(c) Local behaviour: By construction, any point in the set $\mathcal{B}$ defined in the statement of Theorem 1 lies in $\mathcal{X} \times \mathcal{C}_{0}$, because $c_{0}<c_{0}^{\prime}$. We want to prove that any solution pair $((x, \xi),(u, w))$, with $\xi$ initialized in $\mathcal{B}$, experiences no jump on its hybrid time domain, namely $\operatorname{dom}(x, \xi)=[0, \infty) \times\{0\}$. For this, we proceed by contradiction and we assume that there exists $t_{1}>0$ such that $\left(\bar{t}_{1}, 0\right),\left(\bar{t}_{1}, 1\right) \in \operatorname{dom}(x, \xi)$, namely $\xi\left(\bar{t}_{1}, 0\right) \in \mathcal{D}_{0}$. First of all, note that, as $q=0$, the components $\left(x, \zeta_{0}\right)$ of (5) coincide with those of (1) and (2). As a consequence, we can apply Assumption 1. By definition of the set $\mathcal{B}$ defined in the statement of Theorem 1, we have $V_{0}(0,0) \leq \varepsilon_{0}$. As a consequence, item (ii) of Assumption 1 ensures that the solution pair $((x, \xi),(u, w))$ to (5) satisfies $V_{0}(t, 0) \leq \varepsilon_{0}$ for all $t \in\left[0, \bar{t}_{1}\right]$. Then, by applying item (ii) of Assumption 2, we have $\rho_{0}(t, 0) \leq b_{0} c_{0}<b_{0} c_{0}^{\prime}$ for all $t \in\left[0, \bar{t}_{1}\right]$, since $c_{0}<c_{0}^{\prime}$ according to Theorem 1 . Therefore, for $t \in\left[0, \bar{t}_{1}\right]$,

$$
\begin{aligned}
z_{0}(t, 0) & =e^{-b_{0} t} z_{0}(0,0)+\int_{0}^{t} e^{-b_{0}(t-s)} \rho_{0}(s, 0) d s \\
& <e^{-b_{0} t} c_{0}^{\prime}+b_{0}^{-1}\left[1-e^{-b_{0} t}\right] b_{0} c_{0}^{\prime}=c_{0}^{\prime},
\end{aligned}
$$

that is $z_{0}(t, 0)<c_{0}^{\prime}$ for all $t \in\left[0, \bar{t}_{1}\right]$. As a result, $(x, \xi)$ cannot jump at $\left(\bar{t}_{1}, 0\right)$ and we have attained a contradiction. We deduce that $\bar{t}_{1}=\infty$ (recall that any solution is complete) and $\xi(t, 0) \in \mathcal{C}_{0}$ for all $t \in[0, \infty)$. As a result, since the vector field of $\zeta_{0}$ in $\mathcal{C}_{0}$ corresponds to that of the local observer (2), we obtain $\hat{x}=\hat{x}_{0}=\vartheta_{0}\left(\zeta_{0}\right)=\vartheta_{0}\left(\bar{\zeta}_{0}\right)$ on $[0, \infty) \times\{0\}$ where $\bar{\zeta}_{0}$ is a solution to (2) obtained by picking the same initial conditions for (1), (2) and by applying input $(u, w)$ for all time. This concludes the local property and the proof of Theorem 1.

\section{B. Proofs of the claims}

1) Proof of Claim 1: We proceed by contradiction. Let $((x, \xi),(u, w))$ be a solution pair to (5), with $F, G, H, \mathcal{C}, \mathcal{D}$ selected as in (11). For the purpose of showing contradiction, assume that there exists a non-decreasing sequence of hybrid times $\left(t_{n}, j_{n}\right)_{n \in \mathbb{Z}_{\geq 0}} \in \operatorname{dom}(x, \xi)$, with $\left(t_{n}, j_{n}\right) \in \operatorname{dom}(x, \xi)$ for all $n \in \mathbb{Z}_{\geq 0}$, such that (26) holds. Without loss of generality and to simplify the notation, we assume that there is no jump between two elements of this sequence and that $j_{n}=n$. Due to the expression of the function $G$ and the definitions of the sets $\mathcal{C}$ and $\mathcal{D}$ in (11), we have

$$
\begin{aligned}
& \xi(t, j) \in \mathcal{C}_{0} \quad \forall(t, j) \in\left[t_{2 n}, t_{2 n+1}\right] \times\{2 n\} \\
& \xi\left(t_{2 n+1}, 2 n\right) \in \mathcal{D}_{0} \\
& \xi(t, j) \in \mathcal{C}_{1} \quad \forall(t, j) \in\left[t_{2 n+1}, t_{2 n+2}\right] \times\{2 n+1\} \\
& \xi\left(t_{2 n+2}, 2 n+1\right) \in \mathcal{D}_{1}
\end{aligned}
$$

for all $n \in \mathbb{Z}_{\geq 0}$. Due to the expression of the jump map (10b), the components $\left(\zeta_{1}, z_{1}\right)$ do not change at jumps. Thus, since the dynamics in (10a) corresponds to (3) and (9) during flows, solutions $\left(x(t, j), \zeta_{1}(t, j), z_{1}(t, j)\right)$ to (5) correspond to solutions $\left(x(t), \zeta_{1}(t), z_{1}(t)\right)$ to $(1),(3),(9)$ and therefore we can invoke Assumption 4. As a consequence, for $(t, j) \in$ $\left[t_{2 n+1}, t_{2 n+2}\right] \times\{2 n+1\}$, we have

$$
V_{1}(t, j) \leq a_{1} z_{1}(t, j)+\beta_{1}\left(V_{1}\left(t_{0}, j_{0}\right)+z_{1}\left(t_{0}, j_{0}\right), t\right)+v_{1},
$$

recall that $V_{1}(t, j)=V_{1}\left(x(t, j), \zeta_{1}(t, j)\right)$. Since $\beta_{1}$ is of class $\mathcal{K} \mathcal{L}$, and in view of the choice of $c_{1}^{\prime}$ in the statement of Theorem 1, there exists $N \geq 0$ such that

$$
\beta_{1}\left(V_{1}\left(t_{0}, j_{0}\right)+z_{1}\left(t_{0}, j_{0}\right), t_{2 N+2}\right) \leq \varepsilon_{1}^{\prime}-a_{1} c_{1}^{\prime}-v_{1} .
$$

As a consequence, by recalling that, when entering in $\mathcal{D}_{1}$, we have $z_{1} \leq c_{1}^{\prime}$, we obtain $V_{1}\left(t_{2 N+2}, 2 N+1\right) \leq \varepsilon_{1}^{\prime}$ in view of (27). Since

$$
\zeta_{0}\left(t_{2 N+2}, 2 N+2\right)=\Theta\left(\zeta_{1}\left(t_{2 N+2}, 2 N+2\right)\right),
$$

and by applying Assumption 5, we further get

$$
V_{0}\left(t_{2 N+2}, 2 N+2\right) \leq \varepsilon_{0}
$$

It follows from item (ii) of Assumption 1 that $V_{0}(t, j) \leq \varepsilon_{0}$ for all $t \in\left[t_{2 N+2}, t_{2 N+3}\right]$. Then, we can use item (ii) of Assumption 2, to obtain $\rho_{0}(t, 2 N+2) \leq b_{0} c_{0}$ for all 
$t \in\left[t_{2 N+2}, t_{2 N+3}\right]$. As a consequence, by evaluating $z_{0}(t, j)$ at $\left(t_{2 N+3}, 2 N+2\right)$, we have

$$
\begin{aligned}
z_{0}\left(t_{2 N+3}, 2 N+2\right)= & e^{-b_{0}\left(t_{2 N+3}-t_{2 N+2}\right)} z_{0}\left(t_{2 N+2}, 2 N+2\right) \\
& +\int_{t_{2 N+2}}^{t_{2 N+3}} e^{-b_{0}(t-s)} \rho_{0}(s, 2 N+2) d s \\
\leq & 0+b_{0}^{-1} \sup _{t \in\left[t_{2 N+3}-t_{2 N+2}\right]} \rho_{0}(t, 2 N+2)
\end{aligned}
$$

which implies $z_{0}\left(t_{2 N+3}, 2 N+2\right) \leq c_{0}<c_{0}^{\prime}$. The last inequality contradicts $z_{0}\left(t_{2 N+3}, 2 N+2\right) \geq c_{0}^{\prime}$, namely $\xi\left(t_{2 N+3}, 2 N+\right.$ 2) $\in \mathcal{D}_{0}$, and concludes the proof of Claim 1 .

2) Proof of Claim 2: We proceed by contradiction. Consider a solution pair $((x, \xi),(u, w))$ to $(5)$, with $F, G, H, \mathcal{C}, \mathcal{D}$ selected as in (11), such that $q(t, j)=1$ for all $(t, j) \in$ $\operatorname{dom}(x, \xi)$. Since solutions are complete, its time domain is $\operatorname{dom}(x, \xi)=[0, \infty) \times\{0\}, \xi \in \mathcal{C}_{1}$ for all $[0, \infty) \times\{0\}$, and moreover the $\left(\zeta_{0}, \zeta_{1}, z_{0}, z_{1}\right)$ dynamics are

$$
\begin{array}{ll}
\dot{\zeta}_{0}=0, & \dot{\zeta}_{1}=\varphi_{1}\left(\zeta_{1}, u, y\right), \\
\dot{z}_{0}=0, & \dot{z}_{1}=-b_{1} z_{1}+\rho_{1}\left(y, \hat{y}_{1}\right) .
\end{array}
$$

Thus, by applying Assumption 3, we know there exists a $t_{1}>$ 0 such that $V_{1}(t, 0) \leq \varepsilon_{1}$ for all $t \in\left[t_{1}, \infty\right)$. Now consider $\xi$ on $\left[t_{1}, \infty\right)$. By using item (ii) of Assumption 4, we have $\rho_{1}(t, 0) \leq b_{1} c_{1}$ for all $t \in\left[t_{1}, \infty\right)$. Therefore

$$
\begin{aligned}
z_{1}(t, 0) & \leq z_{1}\left(t_{1}, 0\right) e^{-b_{1}\left(t-t_{1}\right)}+b_{1}^{-1} \sup _{s \in\left[t_{1}, t\right]} \rho_{1}(s, 0) \\
& \leq z_{1}\left(t_{1}, 0\right) e^{-b_{1}\left(t-t_{1}\right)}+c_{1}
\end{aligned}
$$

for all $t \in\left[t_{1}, \infty\right)$. Therefore, there exists $t_{2} \in\left[t_{1}, \infty\right)$ such that

$$
z_{1}\left(t_{1}, 0\right) e^{-\lambda_{1}\left(t_{2}-t_{1}\right)}<c_{1}^{\prime}-c_{1} .
$$

Pick any $\bar{t} \geq \max \left\{t_{2}, T\right\}$. As a consequence, $z_{1}(\bar{t}, 0)<c_{1}^{\prime}$, and therefore $\xi(\bar{t}, 0) \notin \mathcal{C}_{1}$ by definition of the map $\mathcal{C}_{1}$ in (11b). This contradicts $q(t, j)=1$ for all $(t, j) \in \operatorname{dom}(x, \xi)$, and concludes the proof of the claim.

3) Proof of Claim 3: Let $((x, \xi),(u, w))$ be a solution pair to (5), with $F, G, H, \mathcal{C}, \mathcal{D}$ selected as in (11), such that there exists $(\bar{t}, \bar{j}) \in \operatorname{dom}(x, \xi)$ with $q(t, j)=0$ for all $(t, j) \succeq(\bar{t}, \bar{j})$, namely $\xi(t, j) \in \mathcal{C}_{0}$ for all $(t, j) \succeq(\bar{t}, \bar{j})$. When flowing in $\mathcal{C}_{0}$, the dynamics of (5) are given by

$$
\begin{array}{ll}
\dot{\zeta}_{0}=\varphi_{0}\left(\zeta_{0}, u, y\right), & \dot{\zeta}_{1}=\varphi_{1}\left(\zeta_{1}, u, y\right), \\
\dot{z}_{0}=-b_{0} z_{0}+\rho_{0}\left(y, h\left(\hat{x}_{0}\right)\right), & \dot{z}_{1}=-b_{1} z_{1}+\rho_{1}\left(y, h\left(\hat{x}_{1}\right)\right) .
\end{array}
$$

namely the dynamics of $\zeta_{0}$ and $z_{0}$ coincide with those of (2) and (7). Therefore we can use Assumptions 1 and 2. Since $q(t, j)=0$ for all $(t, j) \succeq(\bar{t}, \bar{j})$, we also have $z_{0}(t, j) \leq c_{0}^{\prime}$ for all $(t, j) \succeq(\bar{t}, \bar{j})$. By applying item (i) of Assumption 2, we deduce

$$
V_{0}(t, j) \leq a_{0} c_{0}^{\prime}+\beta_{0}\left(V_{0}(\bar{t}, \bar{j})+z_{0}(\bar{t}, \bar{j}), t-\bar{t}\right)+v_{0}
$$

for all $(t, j) \succeq(\bar{t}, \bar{j})$. Recall that $V_{0}(t, j)=$ $V_{0}\left(x(t, j), \zeta_{0}(t, j)\right)$. Since $\beta_{0}$ is a class- $\mathcal{K} \mathcal{L}$ function, and in view of the definition of $c_{0}^{\prime}$ in the statement of the Theorem, there exists $t_{1} \geq \bar{t}$ such that

$$
\beta_{0}\left(V_{0}(\bar{t}, \bar{j})+z_{0}(\bar{t}, \bar{j}), t_{1}-\bar{t}\right) \in\left[0, \varepsilon_{0}^{\prime}-a_{0} c_{0}^{\prime}-v_{0}\right] .
$$

As a consequence, in view of the previous inequality on $V_{0}(t, j)$, we obtain $V_{0}\left(t_{1}, \bar{j}\right) \leq \varepsilon_{0}^{\prime}$. Finally, by applying item (i) of Assumption 1, we conclude that limit (6) holds. The proof of the claim is completed by recalling that $\hat{x}=\hat{x}_{0}$ for $\xi \in \mathcal{C}_{0}$.

\section{Sketch of the Proof of Theorem 2}

We present a sketch of the proof of Theorem 2 as most of the arguments are derived from the proof of Theorem 1.

(a) Completeness of solutions and finite number of jumps: The proof follows the same arguments used in the proof of Theorem 1, see Section VI-A. For this, note that Claim 1 holds also for system (5) with $F$ selected as in (12). The proof is given in Section VI-D1.

(b) Global convergence: The global convergence property can be proved by following the same arguments used in the proof of Theorem 1, see Section VI-A. For this, note that Claims 2 and 3 hold also for system (5) with $F$ selected as in (12). The proof of Claim 2 follows the same arguments used in Section VI-B2 and will therefore not be repeated for the sake of compactness, while the proof of Claim 3 is given in Section VI-D2.

(c) Local behaviour: The proof follows by slightly adaptive the same arguments used in the proof of Theorem 1, see Section VI-A. In particular, we proceed by contradiction and we assume that there exists $\bar{t}>0$ such that $\left(\bar{t}_{1}, 0\right),\left(\bar{t}_{1}, 1\right) \in$ $\operatorname{dom}(x, \xi)$, namely $\xi\left(\bar{t}_{1}, 0\right) \in \mathcal{D}_{0}$. By definition of the set $\mathcal{B}$ defined in the statement of Theorem 2 , we have $V_{0}(0,0) \leq \varepsilon_{0}$ and $V_{1}(0,0) \leq \varepsilon_{1}$. Therefore, in view of item (ii) of Assumption 1 and Assumption 6, we also get $V_{0}(t, 0) \leq \varepsilon_{0}$ and $V_{1}(t, 0) \leq \varepsilon_{1}^{\prime}$ for all $t \in\left[0, \bar{t}_{1}\right]$. By applying item (i) of Assumption 7 , we derive that $\omega(t, 0) \leq c_{0}$ for all $t \in\left[0, \bar{t}_{1}\right]$. Therefore, on $\left[0, \bar{t}_{1}\right]$, the solution $z_{0}$ in (12) satisfies

$$
\begin{aligned}
z_{0}(t, 0) & =e^{-t} z_{0}(0,0)+\int_{0}^{t} e^{-(t-s)} \omega(s, 0) d s \\
& \leq e^{-t} c_{0}+\left[1-e^{-t}\right] c_{0}=c_{0} .
\end{aligned}
$$

Hence $z_{0}(t, 0)<c_{0}^{\prime}$ for all $t \in\left[0, \bar{t}_{1}\right]$. As a result, $z_{0}(t, 0)$ cannot jump at $\left(\bar{t}_{1}, 0\right)$ and we have attained a contradiction. Therefore $\bar{t}_{1}=\infty$ (recall that any solution is complete) and $\xi(t, 0) \in \mathcal{C}_{0}$ for all $t \in[0, \infty)$. The proof concludes with the same arguments of those used in the proof of Theorem 1.

\section{Proof of the claims with F given by (12)}

1) Proof of Claim 1 with F given by (12): As in Section VI-B1, we proceed by contradiction by assuming the existence of a non-decreasing sequence of hybrid times $\left(t_{n}, j_{n}\right)_{n \in \mathbb{Z}_{\geq 0}} \in \operatorname{dom}(x, \xi)$, with $\left(t_{n}, j_{n}\right) \in \operatorname{dom}(x, \xi)$ for all $n \in \mathbb{Z}_{\geq 0}$, such that (26) holds for all $n \in \mathbb{Z}_{\geq 0}$. First of all, by definition of the map $F$ in (12), we see that during flows, the $\zeta_{1}$ dynamics corresponds to that of (3). Moreover, in view of $G$ of (11d), $\zeta_{1}$ has the same value after a jump. As a consequence, Assumption 3 ensures existence of $N \geq 0$ such that $V_{1}\left(t_{2 N+1}, 2 N+1\right) \leq \varepsilon_{1}$. In view of Assumption 6, we further obtain $V_{1}\left(t_{2 N+2}, 2 N+1\right) \leq \varepsilon_{1}^{\prime}$. Therefore, at time $\left(t_{2 N+2}, 2 N+2\right)$,

$$
V_{0}\left(t_{2 N+2}, 2 N+2\right) \leq \varepsilon_{0},
$$


where we used the fact that

$$
\zeta_{0}\left(t_{2 N+2}, 2 N+2\right)=\Theta\left(\zeta_{1}\left(t_{2 N+2}, 2 N+2\right)\right)
$$

in view of the definition of $G$ in (11d), and we applied Assumption 5. As a consequence, by using again Assumption 6 and item (ii) of Assumption 1, we derive $V_{1}(t, 2 N+2) \leq \varepsilon_{1}^{\prime}$ and $V_{0}(t, 2 N+2) \leq \varepsilon_{0}$ for all $t \in\left[t_{2 N+2}, t_{2 N+3}\right]$. The latter implies that $\omega(t, 2 N+2) \leq c_{0}$ for all $t \in\left[t_{2 N+2}, t_{2 N+3}\right]$, in view of item (i) of Assumption 7. As a consequence, by evaluating $z_{0}(t, j)$, and by recalling that $z\left(t_{2 N+2}, 2 N+2\right)=0$ in view of $G$ defined in (11d),

$$
\begin{aligned}
z_{0}\left(t_{2 N+3}, 2 N+2\right) & =\int_{t_{2 N+2}}^{t_{2 N+3}} e^{-(t-s)} \omega(s, 2 N+2) d s \\
& \leq \sup _{t \in\left[t_{2 N+3}-t_{2 N+2}\right]} \omega(t, 2 N+2) \leq c_{0} .
\end{aligned}
$$

Since $c_{0}<c_{0}^{\prime}$, the latter inequality contradicts $\xi\left(t_{2 N+3}, 2 N+\right.$ 2) $\in \mathcal{D}_{0}$ and concludes the proof of the Claim 1 .

2) Proof of Claim 3 with $F$ given by (12): Let $((x, \xi),(u, w))$ be a solution pair to (5) such that there exists $(\bar{t}, \bar{j}) \in \operatorname{dom}(x, \xi), q(t, j)=0$ for all $(t, j) \succeq(\bar{t}, \bar{j})$, namely $\xi(t, j) \in \mathcal{C}_{0}$ for all $(t, j) \succeq(\bar{t}, \bar{j})$. Suppose then, without loss of generality, that $(\bar{t}, \bar{j})=(0,0)$. In view of Assumption 3 and 6 , there exists $t_{0}<\infty$ such that $V_{1}(t, 0) \leq \varepsilon_{1}^{\prime}$ for all $t \in\left[t_{0}, \infty\right)$. Without loss of generality, we assume that $t_{0}=0$. When flowing in $\mathcal{C}_{0}$, the $z_{0}$-dynamics is given by

$$
z_{0}(t, 0)=e^{-t} z_{0}(0,0)+\int_{0}^{t} e^{-(s-t)} \omega(s, 0) d s .
$$

Since $z_{0}(t, 0) \in \mathcal{C}_{0}$ for all for all $t \in[0, \infty)$, it must satisfy $z_{0}(t, 0) \leq c_{0}^{\prime}$ for all $t \in[0, \infty)$, which implies

$$
\int_{0}^{t} e^{-(s-t)} \omega(s, 0) d s \leq c_{0}^{\prime} .
$$

Suppose now that there exists $t_{1}>0$ such that

$$
\omega\left(t_{1}, 0\right) \leq c_{0}^{\prime}+\frac{\left(c_{0}^{\prime \prime}-c_{0}^{\prime}\right)}{2}<c_{0}^{\prime \prime} .
$$

Then, by using item (ii) of Assumption 7, we know $V_{0}\left(t_{1}, 0\right) \leq$ $\varepsilon_{0}^{\prime}$. We conclude, in view of item (i) of Assumption 1, that the limit (6) holds. Consider now the opposite case in which

$$
\omega(t, 0) \geq c_{0}^{\prime}+\frac{\left(c_{0}^{\prime \prime}-c_{0}^{\prime}\right)}{2} \quad \forall t \in[0, \infty) .
$$

As a consequence, in view of (28),

$$
\begin{aligned}
c_{0}^{\prime} & \geq \int_{0}^{t} e^{-(s-t)} \omega(s, 0) d s \geq \int_{0}^{t} e^{-(s-t)}\left(c_{0}^{\prime}+\frac{\left(c_{0}^{\prime \prime}-c_{0}^{\prime}\right)}{2}\right) d s \\
& \geq\left(c_{0}^{\prime}+\frac{\left(c_{0}^{\prime \prime}-c_{0}^{\prime}\right)}{2}\right)\left(1-e^{-t}\right)
\end{aligned}
$$

which implies

$$
e^{-t} \geq \frac{c_{0}^{\prime \prime}-c_{0}^{\prime}}{c_{0}^{\prime \prime}+c_{0}^{\prime}}>0
$$

for all $t \in[0, \infty)$, which cannot hold since $\lim _{t \rightarrow \infty} e^{-t}=0$. We deduce that the solution $(x, \xi)$ cannot satisfy at the same time (28) and (29) for all $t \in[0, \infty)$. In particular, either a jump occur (contradicting our assumptions), either there must exist $t_{1} \in[0, \infty)$ such that

$$
\omega\left(t_{2}, 0\right) \leq c_{0}^{\prime}+\frac{\left(c_{0}^{\prime \prime}-c_{0}^{\prime}\right)}{2}
$$

for all $t \in\left[t_{1}, \infty\right)$, which concludes the proof.

\section{E. Proof of Proposition 1}

We prove the result of the proposition by verifying each assumption. Note that Assumption 1 is automatically implied by Assumption 8 .

Assumption 2. Let $\Phi_{0}\left(x, u, w, \zeta_{0}\right)=\left(f(x, u), f\left(\hat{x}_{0}, u\right)+\right.$ $K_{0}\left(\zeta_{0}\right)\left(h(x, w)-h\left(\hat{x}_{0}, 0\right)\right)$. By using the properties of boundedness of $K_{0}, K_{1}$, we obtain

$$
\begin{aligned}
\mid \Phi_{0}\left(x, u, w, \zeta_{0}\right) & -\Phi_{1}\left(x, u, w, \zeta_{0}\right) \mid \\
\leq & \left|K_{0}\left(\zeta_{0}\right)\left(y-\hat{y}_{0}\right)-K_{1}\left(\hat{x}_{1}\right)\left(y-\hat{y}_{0}\right)\right| \\
\leq & \left(k_{0}+k_{1}\right)\left|y-\hat{y}_{0}\right| .
\end{aligned}
$$

Next, by using the function $V_{1}$ defined in Assumption 9, the previous inequality and the second inequality in (16), we compute

$$
\begin{aligned}
& \left\langle\nabla V_{1}\left(x, \hat{x}_{0}\right), \Phi_{0}\left(x, u, w, \hat{x}_{0}\right)\right\rangle \\
& =\left\langle\nabla V_{1}\left(x, \hat{x}_{0}\right), \Phi_{1}\left(x, u, w, \hat{x}_{0}\right)\right\rangle \\
& \quad+\left\langle\nabla V_{1}\left(x, \hat{x}_{0}\right), \Phi_{0}\left(x, u, w, \hat{x}_{0}\right)-\Phi_{1}\left(x, u, w, \hat{x}_{0}\right)\right\rangle \\
& \quad \leq-\lambda_{1} V_{1}\left(x, \hat{x}_{0}\right)+\psi_{1}(|w|)+k_{2}\left|x-\hat{x}_{0}\right|\left(k_{0}+k_{1}\right)\left|y-\hat{y}_{0}\right|
\end{aligned}
$$

for any $\left(x, \zeta_{0}\right) \in \mathcal{X} \times \mathcal{Z}_{0}$ and $(u, w) \in \mathcal{U} \times \mathcal{W}$. Furthermore, by using the next inequality, obtained by combining Young's inequality, the first inequality in (16) and the definition of $\varrho_{0}$ given in Table I,

$$
\begin{aligned}
k_{2} \mid x & -\hat{x}_{0}\left|\left(k_{0}+k_{1}\right)\right| y-\hat{y}_{0} \mid \\
& \leq \frac{1}{2} \lambda_{1} \underline{\alpha}_{1}\left|x-\hat{x}_{0}\right|^{2}+\frac{1}{2} k_{2}^{2}\left(k_{0}+k_{1}\right)^{2}\left(\lambda_{1} \underline{\alpha}_{1}\right)^{-1}\left|y-\hat{y}_{0}\right|^{2} \\
& \leq \frac{1}{2} \lambda_{1} V_{1}\left(x, \hat{x}_{0}\right)+\varrho_{0}\left|y-\hat{y}_{0}\right|^{2},
\end{aligned}
$$

we finally obtain

$$
\begin{aligned}
\left\langle\nabla V_{1}\left(x, \hat{x}_{0}\right),\right. & \left.\Phi_{0}\left(x, u, w, \hat{x}_{0}\right)\right\rangle \\
& \leq-b_{0} V_{1}\left(x, \hat{x}_{0}\right)+\psi_{1}(|w|)+\varrho_{0}\left|y-\hat{y}_{0}\right|^{2}
\end{aligned}
$$

with $b_{0}$ given in Table I. By letting (7) be defined with $\rho_{0}$ defined as in Table I, we derive, using the comparison principle, that

$$
\begin{aligned}
V_{1}\left(x(t), \hat{x}_{0}(t)\right) \leq & z_{0}(t)+b_{0}^{-1} \psi_{1}(|\bar{w}|) \\
& +e^{-b_{0} t}\left(V_{1}\left(x(0), \hat{x}_{0}(0)\right)+z_{0}(0)\right)
\end{aligned}
$$

for all $t \geq 0$. Furthermore, in view of Assumptions 8-9,

$$
\begin{aligned}
\frac{1}{\bar{p}_{0}}\left|x-\hat{x}_{0}\right|^{2} \leq V_{0}\left(x, \zeta_{0}\right) & \leq \frac{1}{\underline{p}_{0}}\left|x-\hat{x}_{0}\right|^{2} \\
\frac{1}{\bar{p}_{0} \bar{\alpha}_{1}} V_{1}\left(x, \hat{x}_{0}\right) \leq V_{0}\left(x, \zeta_{0}\right) & \leq \frac{1}{\underline{p}_{0} \underline{\alpha}_{1}} V_{1}\left(x, \hat{x}_{0}\right)
\end{aligned}
$$

for any $\left(x, \zeta_{0}\right) \in \mathcal{X} \times \mathcal{Z}_{0}$. By using (30) and (31), we deduce that item (i) of Assumption 2 holds with $a_{0}, v_{0}$ given in Table I, and, for any $s, t \geq 0$,

$$
\beta_{0}(s, t):=\left(\underline{p}_{0} \underline{\alpha}_{1}\right)^{-1} \max \left\{1, \bar{p}_{0} \bar{\alpha}_{1}\right\} e^{-b_{0} t} s .
$$

Now let us introduce the following compact notation

$$
\sup _{\varepsilon_{0}, \bar{w}} \rho_{0}\left(y, \hat{y}_{0}\right):=\sup \left\{\varrho_{0}\left|y-\hat{y}_{0}\right|^{2}:\left(x, \zeta_{0}\right) \in \mathcal{X} \times \mathcal{Z}_{0},\right.
$$$$
\left.V_{0}\left(x, \zeta_{0}\right) \leq \varepsilon_{0}, w \in \mathcal{W}\right\} \text {. }
$$ 
By using (13), (31) and the parameters $\mu_{0}, \mu_{0}^{\prime}$ and $c_{0}$ defined in Table I, item (ii) of Assumption 2 is satisfied by computing

$$
\begin{gathered}
\sup _{\varepsilon_{0}, \bar{w}} \rho_{0}\left(y, \hat{y}_{0}\right) \leq \varrho_{0}\left(L_{x} \sup _{V_{0}\left(x, \zeta_{0}\right) \leq \varepsilon_{0}}\left|x-\hat{x}_{0}\right|+L_{w} \bar{w}\right)^{2} \\
\leq \varrho_{0}\left(2 L_{x}^{2} \bar{p}_{0} \varepsilon_{0}+2 L_{w}^{2} \bar{w}^{2}\right)=\mu_{0} \varepsilon_{0}+\mu_{0}^{\prime} \bar{w}^{2} \leq b_{0} c_{0} .
\end{gathered}
$$

Furthermore, by definition of $c_{0}$, we have $a_{0} c_{0} \geq \varepsilon_{0}$. This shows the lower bound of the inequality of item (iii) of Assumption 2. Finally, by using the condition (17b), and the definition of $c_{0}$ in Table I, we directly obtain $\varepsilon_{0}^{\prime}>a_{0} c_{0}+v_{0}$ by which we obtain the upper bound of the inequality of item (iii) of Assumption 2.

Assumption 3. By using Assumption 9 we can show that all solutions to (1), (15) converge to the set

$$
\left\{\left(x, \zeta_{1}\right): V_{1}\left(x, \zeta_{1}\right) \leq v_{1}\right\},
$$

with $v_{1}$ defined in Table I. The result follows selecting $\varepsilon_{1}>v_{1}$ according to Table I.

Assumption 4. By using the comparison principle to the inequality obtained by subtracting (9), with $a_{1}, b_{1}$ and $\rho_{1}$ selected as in Table I, to the last inequality of (16), we can deduce item (i) of Assumption 4, in which, for any $s, t \geq 0$, $\beta_{1}(s, t):=e^{-b_{1} t} s$ and $v_{1}$ selected as in Table I. Now let us define the compact notation

$$
\begin{array}{r}
\sup _{\varepsilon_{1}, \bar{w}} \rho_{1}\left(y, \hat{y}_{1}\right):=\sup \left\{\varrho_{1}\left|y-\hat{y}_{1}\right|^{2}:\left(x, \zeta_{1}\right) \in \mathcal{X} \times \mathcal{Z}_{1},\right. \\
\left.V_{1}\left(x, \zeta_{1}\right) \leq \varepsilon_{1}, w \in \mathcal{W}\right\} .
\end{array}
$$

By using (13), the first inequality in (16), and the definition of $\varrho_{1}$ in Table I, we compute

$$
\begin{aligned}
\sup _{\varepsilon_{1}, \bar{w}} \rho_{1}\left(y, \hat{y}_{1}\right) & \leq \varrho_{1}\left(L_{x} \sup _{V_{1}\left(x, \zeta_{1}\right) \leq \varepsilon_{1}}\left|x-\hat{x}_{1}\right|+L_{w} \bar{w}\right)^{2} \\
& \leq \varrho_{1}\left(L_{x} \sqrt{\varepsilon_{1} \underline{\alpha}_{1}^{-1}}+L_{w} \bar{w}\right)^{2} \leq b_{1} c_{1} .
\end{aligned}
$$

This shows item (ii) of Assumption 4. By using the definitions of $a_{1}, c_{1}, \varepsilon_{1}, \varepsilon_{1}^{\prime}, v_{1}$ in Table I, we have $\varepsilon_{1}=a_{1} c_{1}<\varepsilon_{1}^{\prime}-v_{1}$ showing the inequality in in item (iii) of Assumption 4. Note that condition (17a) and the choice of $\varepsilon_{1}^{\prime}$ implies $\varepsilon_{1}^{\prime}>2 v_{1}$, thus ensuring that the set $\left(v_{1}, \varepsilon_{1}^{\prime}-v_{1}\right)$ is non empty.

Assumption 5. Let us define the compact notation

$$
\begin{array}{r}
\sup _{\varepsilon_{1}^{\prime}} V_{0}\left(x, \zeta_{0}\right):=\sup \left\{V_{0}\left(x, \zeta_{0}\right):\left(x, \zeta_{1}\right) \in \mathcal{X} \times \mathcal{Z}_{1},\right. \\
\left.\zeta_{0}=\Theta\left(\zeta_{1}\right), V_{1}\left(x, \zeta_{1}\right) \leq \varepsilon_{1}^{\prime}\right\} .
\end{array}
$$

By using (31), and the definition of $\varepsilon_{1}^{\prime}$ in Table I, we compute

$$
\sup _{\varepsilon_{1}^{\prime}} V_{0}\left(x, \zeta_{0}\right) \leq \frac{1}{\bar{p}_{0}} \sup _{V_{1}\left(x, \zeta_{1}\right) \leq \varepsilon_{1}^{\prime}}\left|x-\hat{x}_{1}\right|^{2} \leq \frac{\varepsilon_{1}^{\prime}}{\underline{\alpha}_{1} \bar{p}_{0}}=\varepsilon_{0},
$$

which concludes the proof.

\section{F. Proof of Proposition 2}

We prove the result of the proposition by verifying each assumption. Note that Assumption 1 is automatically implied by Assumption 8 .

Assumption 3. By combining (13) and (21), we obtain

$$
\begin{aligned}
\left|h(x, w)-h_{a}(x)\right| & =\left|h(x, w)-h(x, 0)+h(x, 0)-h_{a}(x)\right| \\
& \leq L_{w} \bar{w}+\Delta_{h}
\end{aligned}
$$

for all $x \in \mathcal{X}$ and $w \in \mathcal{W}$. Let $\Phi_{1}\left(x, u, w, \hat{x}_{1}\right):=$ $\left(f(x, u), f_{a}\left(\hat{x}_{1}, u\right)+K_{1}\left(\hat{x}_{1}\right)\left(h(x, w)-h_{a}\left(\hat{x}_{1}\right)\right)\right.$. By using the previous inequality, by recalling the definition of $\Phi_{a}$ given in Assumption 10, by using the property of $K_{1}$ and inequality (21), we compute

$$
\begin{aligned}
& \left|\Phi_{1}\left(x, u, w, \hat{x}_{1}\right)-\Phi_{a}\left(x, u, \hat{x}_{1}\right)\right| \\
& \quad \leq\left|f(x, u)-f_{a}(x, u)\right|+\left|K_{1}\left(\hat{x}_{1}\right)\left(h(x, w)-h_{a}(x)\right)\right| \\
& \quad \leq \Delta_{f}+k_{1}\left(L_{w} \bar{w}+\Delta_{h}\right) .
\end{aligned}
$$

As a consequence, by using the function $V_{1}$ given in Assumption 10 , we obtain

$$
\begin{aligned}
\langle\nabla & \left.V_{1}\left(x, \hat{x}_{1}\right), \Phi_{1}\left(x, u, w, \hat{x}_{1}\right)\right\rangle \\
= & \left\langle\nabla V_{1}\left(x, \hat{x}_{1}\right), \Phi_{a}\left(x, u, \hat{x}_{1}\right)\right\rangle \\
& +\left\langle\nabla V_{1}\left(x, \hat{x}_{1}\right), \Phi_{1}\left(x, u, w, \hat{x}_{1}\right)-\Phi_{a}\left(x, u, \hat{x}_{1}\right)\right\rangle \\
\leq & -\lambda_{1} V_{1}\left(x, \hat{x}_{1}\right)+k_{2}\left|x-\hat{x}_{1}\right|\left(\Delta_{f}+k_{1}\left(L_{w} \bar{w}+\Delta_{h}\right)\right) .
\end{aligned}
$$

Next, by using the following inequality, obtained by applying Young's inequality and the first inequality in (20),

$$
\begin{aligned}
& k_{2}\left|x-\hat{x}_{1}\right|\left(\Delta_{f}+k_{1}\left(L_{w} \bar{w}+\Delta_{h}\right)\right) \\
& \quad \leq \frac{1}{2} \lambda_{1} \underline{\alpha}_{1}\left|x-\hat{x}_{1}\right|^{2}+\frac{1}{2}\left(\lambda_{1} \underline{\alpha}_{1}\right)^{-2}\left(\Delta_{f}+k_{1}\left(L_{w} \bar{w}+\Delta_{h}\right)\right)^{2} \\
& \quad \leq b_{1} V_{1}\left(x, \hat{x}_{1}\right)+v_{1}
\end{aligned}
$$

with $b_{1}, v_{1}$ defined as in Table II, we further obtain

$$
\left\langle\nabla V_{1}\left(x, \hat{x}_{1}\right), \Phi_{1}\left(x, u, w, \hat{x}_{1}\right)\right\rangle \leq-b_{1} V_{1}\left(x, \hat{x}_{1}\right)+b_{1} v_{1} .
$$

Finally, by applying the comparison principle to the previous inequality we obtain

$$
V_{1}\left(x(t), \zeta_{1}(t)\right) \leq e^{-b_{1} t} V_{1}\left(x(0), \zeta_{1}(0)\right)+v_{1} .
$$

Assumption 3 is satisfied with the choice $\varepsilon_{1}>v_{1}$ in Table II.

Assumption 4. By subtracting the solution to (9), in which $b_{1}, \rho_{1}$ are selected as in Table II, to inequality in (32), we obtain directly obtain item (i) of Assumption 4, in which $a_{1}, v_{1}$ are defined as in Table II and $\beta_{1}(s, t)=e^{-b_{1} t} s$ for any $s, t \geq$ 0 . By combining (13) and (21), we also obtain

$$
\left|h(x, w)-h_{a}\left(\hat{x}_{1}\right)\right| \leq L_{x}\left|x-\hat{x}_{1}\right|+L_{w} \bar{w}+\Delta_{h} .
$$

Define

$$
\begin{array}{r}
\sup _{\varepsilon_{1}, \bar{w}} \rho_{1}\left(y, \hat{y}_{1}\right):=\sup \left\{\varrho_{1}\left|y-\hat{y}_{1}\right|^{2}:\left(x, \zeta_{1}\right) \in \mathcal{X} \times \mathcal{Z}_{1},\right. \\
\left.V_{1}\left(x, \zeta_{1}\right) \leq \varepsilon_{1}, w \in \mathcal{W}\right\} .
\end{array}
$$

Then, by using the first inequality in (20), we compute

$$
\begin{aligned}
\sup _{\varepsilon_{1}, \bar{w}} \rho_{1}\left(y, \hat{y}_{1}\right) \leq & \varrho_{1}\left(L_{x} \sup _{V_{1}\left(x, \zeta_{1}\right) \leq \varepsilon_{1}}\left|x-\hat{x}_{1}\right|+L_{w} \bar{w}+\Delta_{h}\right)^{2} \\
& \leq \varrho_{1}\left(L_{x} \sqrt{\frac{\varepsilon_{1}}{\underline{\alpha}_{1}}}+L_{w} \bar{w}+\Delta_{h}\right)^{2} \leq b_{1} c_{1}
\end{aligned}
$$

in which we used the definition of $\varrho_{1}$ in Table II to compute the last inequality. This shows item (ii) of Assumption 4 holds. By using the definitions of $a_{1}, c_{1}, v_{1}$ in Table II, we have $a_{1} c_{1}=\varepsilon_{1}$. In view of the choice of $\varepsilon_{1}$ By using the definitions of $a_{1}, c_{1}, \varepsilon_{1}, \varepsilon_{1}^{\prime}, v_{1}$ in Table I, we have $\varepsilon_{1}=a_{1} c_{1}<\varepsilon_{1}^{\prime}-v_{1}$ showing the inequality in in item (iii) of Assumption 4. Note that condition (22) and the choice of $\varepsilon_{1}^{\prime}$ implies $\varepsilon_{1}^{\prime}>2 v_{1}$, thus ensuring that the set $\left(v_{1}, \varepsilon_{1}^{\prime}-v_{1}\right)$ is non empty. 
Assumption 5. Let

$$
\begin{array}{r}
\sup _{\varepsilon_{1}^{\prime}} V_{0}\left(x, \zeta_{0}\right):=\sup \left\{V_{0}\left(x, \zeta_{0}\right):\left(x, \zeta_{1}\right) \in \mathcal{X} \times \mathcal{Z}_{1},\right. \\
\left.\zeta_{0}=\Theta\left(\zeta_{1}\right), V_{1}\left(x, \zeta_{1}\right) \leq \varepsilon_{1}^{\prime}\right\} .
\end{array}
$$

By using (31), and the definition of $\varepsilon_{1}^{\prime}$ in Table II, we compute

$$
\sup _{\varepsilon_{1}^{\prime}} V_{0}\left(x, \zeta_{0}\right) \leq \frac{1}{\bar{p}_{0}} \sup _{V_{1}\left(x, \zeta_{1}\right) \leq \varepsilon_{1}^{\prime}}\left|x-\hat{x}_{1}\right|^{2} \leq \frac{\varepsilon_{1}^{\prime}}{\underline{\alpha}_{1} \bar{p}_{0}}=\varepsilon_{0} .
$$

Assumption 6. Pick any initial condition satisfying $V_{1}\left(x(0), \zeta_{1}(0)\right) \leq \varepsilon_{1}$. By using (32) and the definition of $\varepsilon_{1}$ in Table II we directly obtain

$$
V\left(x(t), \zeta_{1}(t)\right) \leq e^{-b_{1} t} \varepsilon_{1}+v_{1}<\varepsilon_{1}^{\prime}
$$

for all $t \geq 0$.

Assumption 7. First of all, we need to verify that $c_{0}^{\prime \prime}>c_{0}$. For this, by using their definition in Table II, we compute

$$
c_{0}^{\prime \prime}-c_{0}=\frac{1}{2}\left(\underline{p}_{0} \varepsilon_{0}^{\prime}-2 \bar{p}_{0} \varepsilon_{0}\right)-4 \bar{p}_{0} \varepsilon_{0}=\frac{1}{2} \underline{p}_{0}\left(\varepsilon_{0}^{\prime}-10 \frac{\bar{p}_{0}}{\underline{p}_{0}} \varepsilon_{0}\right)
$$

which implies $c_{0}^{\prime \prime}>c_{0}$ in view of the condition (22) in the statement of the proposition. Moreover, the function $\omega$ defined in Table II satisfies the condition $\omega\left(\Theta\left(\zeta_{1}\right), \zeta_{1}\right)=0$ of the statement of Assumption 7. Now we prove items (i), (ii) of Assumption 7. To show item (i), we use the bounds in (31), the first inequality in (20) and following inequality

$$
\frac{1}{2}\left|\hat{x}_{0}-\hat{x}_{1}\right|^{2} \leq\left|x-\hat{x}_{0}\right|^{2}+\left|x-\hat{x}_{1}\right|^{2} .
$$

We obtain

$$
\omega\left(\zeta_{0}, \zeta_{1}\right) \leq 2 \bar{p}_{0} V_{0}\left(x, \zeta_{0}\right)+2 \underline{\alpha}_{1}^{-1} V_{1}\left(x, \zeta_{1}\right)
$$

and therefore, by using the compact notation

$$
\begin{array}{r}
\bar{\omega}:=\sup \left\{\omega\left(\zeta_{0}, \zeta_{1}\right):\left(x, \zeta_{0}, \zeta_{1}\right) \in \mathcal{X} \times \mathcal{Z}_{0} \times \mathcal{Z}_{1},\right. \\
\left.V_{0}\left(x, \zeta_{0}\right) \leq \varepsilon_{0}, V_{1}\left(x, \zeta_{1}\right) \leq \varepsilon_{1}^{\prime}\right\},
\end{array}
$$

and by recalling the definition of $\varepsilon_{1}^{\prime}, c_{0}$ in Table II, we directly obtain

$$
\bar{\omega} \leq 2 \bar{p}_{0} \varepsilon_{0}+2 \underline{\alpha}_{1}^{-1} \varepsilon_{1}^{\prime} \leq 4 \bar{p}_{0} \varepsilon_{0}=c_{0} .
$$

To show item (ii) of Assumption 7, we combine inequality (31), the first inequality in (20), the definition of $\omega\left(\zeta_{0}, \zeta_{1}\right)$ in Table II, and the following inequality

$$
\frac{1}{2}\left|x-\hat{x}_{0}\right|^{2} \leq\left|x-\hat{x}_{1}\right|^{2}+\left|\hat{x}_{1}-\hat{x}_{0}\right|^{2} .
$$

We obtain

$$
V_{0}\left(x, \zeta_{0}\right) \leq 2 \underline{p}_{0}^{-1} \underline{\alpha}_{1}^{-1} V_{1}\left(x, \zeta_{1}\right)+2 \underline{p}_{0}^{-1} \omega\left(\zeta_{0}, \zeta_{1}\right) .
$$

As a consequence, by using the compact notation

$$
\begin{array}{r}
\bar{V}_{0}:=\sup \left\{V_{0}\left(x, \zeta_{0}\right):\left(x, \zeta_{0}, \zeta_{1}\right) \in \mathcal{X} \times \mathcal{Z}_{0} \times \mathcal{Z}_{1},\right. \\
\left.V_{1}\left(x, \zeta_{1}\right) \leq \varepsilon_{1}^{\prime}, \omega\left(\zeta_{0}, \zeta_{1}\right) \leq c_{0}^{\prime \prime}\right\},
\end{array}
$$

and by recalling the definition of $\varepsilon_{1}^{\prime}, c_{0}^{\prime \prime}$ in Table II, we derive $\bar{V}_{0} \leq 2 \underline{p}_{0}^{-1} \underline{\alpha}_{1}^{-1} \varepsilon_{1}^{\prime}+2 \underline{p}_{0}^{-1} c_{0}^{\prime \prime} \leq 2 \underline{p}_{0}^{-1} \bar{p}_{0} \varepsilon_{0}+\varepsilon_{0}^{\prime}-2 \underline{p}_{0}^{-1} \bar{p}_{0} \varepsilon_{0}$ and therefore $\bar{V}_{0} \leq \varepsilon_{0}^{\prime}$. This concludes the proof of the proposition.

\section{CONCLUSION}

We have addressed the problem of combining two given observers, one ensuring global convergence, and the other guaranteeing some desired (possibly optimal) behaviour when the estimation error is small. Under a set of sufficient conditions, we provided a constructive solution based on a hybridredesign and two norm estimators that are used to detect whether the estimation error provided by each observer is small enough. The resulting uniting observer takes benefit of the good properties of each observer: it guarantees the global convergence of the estimation error, while preserving the desired local behaviour, asymptotically. Then, two case studies are proposed. We first combine an EKF with a global asymptotic observer that can be designed using various techniques borrowed from the literature, see e.g., [2], [15], [26], [27]. Then, we study the case in which the global observer is not asymptotically convergent.

We point out that the proposed solution is not unique and depends, in general, on the properties of the given observers. For instance, in the proposed scheme, the global observer is enforced to run also when the current estimate coincides with that of local observer. However, when both observers possess global convergence properties, one can exploit a different design in which only one observer at a time is used, in order to reduce the overall computational cost. This case will be addressed in future works.

The main message of this work, namely to combine different observers in order to improve the overall performance, can be adapted to take into account also different scenarios which have not been explicitly addressed here. For instance, in order to relax the matching conditions of Assumption 5, it may be possible to substitute the local observer by a bank of local observers, in the same spirit of [10], as mentioned in Remark 1. Compared to [10], uniting a single global observer with a bank of local oscillators could be more efficient from the computation point of view. This work also suggests the development of new classes of global approximate/practical observers that would not be of great interest without the unification of a local observer.

\section{REFERENCES}

[1] M. M. Seron, J. H. Braslavsky, and G. C. Goodwin, Fundamental Limitations in Filtering and Control. Springer, 1997.

[2] H. K. Khalil and L. Praly, "High-gain observers in nonlinear feedback control," International Journal of Robust and Nonlinear Control, vol. 24, no. 6, pp. 993-1015, 2014.

[3] Y. Shtessel, C. Edwards, L. Fridman, and A. Levant, Sliding mode control and observation. Birkhäuser, 2014.

[4] S. Bonnabel and J.-J. Slotine, "A contraction theory-based analysis of the stability of the deterministic extended kalman filter," IEEE Transactions on Automatic Control, vol. 60, no. 2, pp. 565-569, 2015.

[5] A. J. Krener, "The convergence of the extended kalman filter," in Directions in mathematical systems theory and optimization. Springer, 2003, vol. 286, pp. 173-182.

[6] R. Sanfelice and L. Praly, "On the performance of high-gain observers with gain adaptation under measurement noise," Automatica, vol. 47, pp. 2165-2176, 2011.

[7] N. Boizot, E. Busvelle, and J.-P. Gauthier, "An adaptive high-gain observer for nonlinear systems," Automatica, vol. 46, no. 9, pp. 1483$1488,2010$.

[8] A. Krener and A. Duarte, "A hybrid computational approach to nonlinear estimation," in 35th IEEE Conference on Decision and Control, vol. 2, 1996, pp. $1815-1819$. 
[9] B. Clement and G. Duc, "An interpolation method for gain-scheduling," in 40th IEEE Conference on Decision and Control, vol. 2, 2001, pp. 1310-1315.

[10] R. Postoyan, M. H. Hamid, and J. Daafouz, "A multi-observer approach for the state estimation of nonlinear systems," in 54th IEEE Conference on Decision and Control, 2015, pp. 1793-1798.

[11] C. Prieur and L. Praly, "Uniting local and global controllers," in 38th IEEE Conference on Decision and Control, vol. 2, 1999, pp. 1214-1219.

[12] C. Prieur and A. R. Teel, "Uniting local and global output feedback controllers," IEEE Transactions on Automatic Control, vol. 56, no. 7, pp. 1636-1649, 2011

[13] A. R. Teel, O. E. Kaiser, and R. M. Murray, "Uniting local and global controllers for the caltech ducted fan," in American Control Conference, vol. 3. IEEE, 1997, pp. 1539-1543.

[14] C. Prieur, "Uniting local and global controllers with robustness to vanishing noise," Mathematics of Control, Signals and Systems, vol. 14, no. 2, pp. 143-172, 2001.

[15] A. Zemouche and M. Boutayeb, "On lmi conditions to design observers for lipschitz nonlinear systems," Automatica, vol. 49, pp. 585-581, 2013.

[16] K. Reif, F. Sonneman, and R. Unbehauen, "An ekf-based nonlinear observer with a prescribed degree of stability," Automatica, vol. 34, pp. $1119-1123,1998$.

[17] R. Rajamani, "Observers for lipschitz nonlinear systems," IEEE transactions on Automatic Control, vol. 43, no. 3, pp. 397-401, 1998.

[18] D. Astolfi and C. Possieri, "Design of local observers for autonomous nonlinear systems not in observability canonical form," Automatica, vol. 103, pp. 443-449, 2019.

[19] M. H. Hamid, R. Postoyan, and J. Daafouz, "Local observers design for a class of neural mass models," in European Control Conference, 2015, pp. $1830-1835$

[20] G. Besançon, Nonlinear observers and applications. Springer, 2007.

[21] P. Bernard, Observer Design for Nonlinear Systems. Springer, 2019.

[22] D. Astolfi and L. Praly, "Integral action in output feedback for multiinput multi-output nonlinear systems," IEEE Transactions on Automatic Control, vol. 62, no. 4, pp. 1559-1574, 2017.

[23] D. Astolfi and L. Marconi, "A high-gain nonlinear observer with limited gain power," IEEE Transactions on Automatic Control, vol. 60, no. 11, pp. 3059-3064, 2015.

[24] V. Andrieu and L. Praly, "On the existence of a kazantziskravaris/luenberger observer," SIAM Journal on Control and Optimization, vol. 45, no. 2, pp. 432-456, 2006.

[25] L. Wang, D. Astolfi, L. Marconi, and H. Su, "High-gain observers with limited gain power for systems with observability canonical form," Automatica, vol. 75, pp. 16-23, 2017.

[26] M. Arcak and P. Kokotović, "Nonlinear observers: a circle criterion design and robustness analysis," Automatica, vol. 37, no. 12, pp. $1923-$ 1930, 2001

[27] D. Astolfi, L. Marconi, L. Praly, and A. R. Teel, "Low-power peakingfree high-gain observers," Automatica, vol. 98, pp. 169-179, 2018.

[28] M. Krichman, E. D. Sontag, and Y. Wang, "Input-output-to-state stability," SIAM Journal on Control and Optimization, vol. 39, no. 6, pp. 1874-1928, 2001.

[29] J.-P. Gauthier and I. Kupka, Deterministic observation theory and applications. Cambridge university press, 2001.

[30] D. Astolfi, R. Postoyan, and D. Nešić, "Uniting local and global observers for the state estimation of nonlinear continuous-time systems," in IEEE 56th Annual Conference on Decision and Control, 2017, pp. 3039-3044.

[31] C. Cai and A. R. Teel, "Characterizations of input-to-state stability for hybrid systems," Systems \& Control Letters, vol. 58, no. 1, pp. 47-53, 2009.

[32] R. Goebel, R. Sanfelice, and A. R. Teel, Hybrid dynamical systems. Princeton, 2011.

[33] H. Shim, J. H. Seo, and A. Teel, "Nonlinear observer design via passivation of error dynamics," Automatica, vol. 39, pp. 885-892, 2003.

[34] D. Efimov, T. Raïssi, S. Chebotarev, and A. Zolghadri, "Interval state observer for nonlinear time varying systems," Automatica, vol. 49, no. 1, pp. 200-205, 2013.

[35] E. D. Sontag and Y. Wang, "Output-to-state stability and detectability of nonlinear systems," Systems and Control Letters, vol. 29, pp. 279-290, 1997.

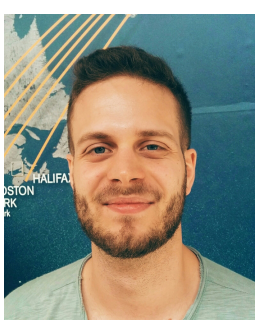

Daniele Astolfi received the B.S. and M.S. degrees in automation engineering from the University of Bologna, Italy, in 2009 and 2012, respectively. He obtained a joint $\mathrm{Ph} . \mathrm{D}$. degree in Control Theory from the University of Bologna, Italy, and from Mines ParisTech, France, in 2016.

In 2016 and 2017, he has been a Research Assistant at the University of Lorraine (CRAN), Nancy, France. Since 2018, he is a CNRS Researcher at LAGEPP, Lyon, France.

His research interests include observer design, feedback stabilization and output regulation for nonlinear systems, networked control systems, hybrid systems, and multi-agent systems.

He serves as an associate editor of the IFAC journal Automatica. He was a recipient of the 2016 Best Italian Ph.D. Thesis Award in Automatica given by SIDRA and nominated for the Best Student Paper Award at ECC 2016.

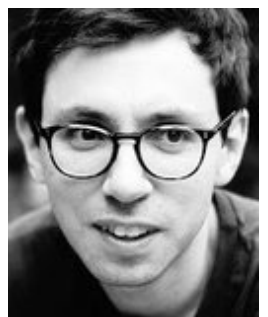

Romain Postoyan received the M.Sc. degree in Electrical and Control Engineering from ENSEEIHT (France) in 2005. He obtained the M.Sc. by Research in Control Theory \& Application from Coventry University (United Kingdom) in 2006 and the $\mathrm{Ph} . \mathrm{D}$. in Control Theory from Université Paris-Sud (France) in 2009

In 2010, he was a research assistant at the University of Melbourne (Australia). Since 2011, he is a CNRS researcher at the Centre de Recherche en Automatique de Nancy (France).

He serves/served as an associate editor for the journals: Automatica, IEEE Control Systems Letters and IMA Journal of Mathematical Control and Information.

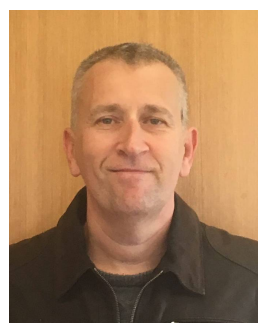

Dragan Nešić is a Professor in the Department of Electrical and Electronic Engineering (DEEE) at The University of Melbourne, Australia. He received his BE degree in Mechanical Engineering from The University of Belgrade, Yugoslavia in 1990, and his $\mathrm{Ph} . \mathrm{D}$. degree from Systems Engineering, RSISE Australian National University, Canberra, Australia in 1997.

Since February 1999 he has been with The University of Melbourne. His research interests include networked control systems, reset systems, extremum seeking control, hybrid control systems, and so on.

He was a co-receipient of the George S. Axelby Outstanding Paper Award (2018). He was also awarded a Humboldt Research Fellowship (2003) by the Alexander von Humboldt Foundation, an Australian Professorial Fellowship (2004-2009) and Future Fellowship (2010-2014) by the Australian Research Council. He is a Fellow of IEEE. He served as a Distinguished Lecturer of CSS, IEEE and as a Member of the Board of Governors, CSS, IEEE. He also served as an Associate Editor for the journals Automatica, IEEE Transactions on Automatic Control, Systems and Control Letters, European Journal of Control and as a General Co-Chair of IEEE CDC 2017 in Melbourne. Currently, he serves as an Associate Editor for the IEEE Transactions on Control of Network Systems (CONES). 